\title{
Symmetry, quantitative Liouville theorems and analysis of large solutions of conformally invariant fully nonlinear elliptic equations
}

\author{
YanYan $\mathrm{Li}^{1}$ • Luc Nguyen ${ }^{2}$
}

Received: 9 February 2017 / Accepted: 4 May 2017 / Published online: 19 June 2017

(C) The Author(s) 2017. This article is an open access publication

\begin{abstract}
We establish blow-up profiles for any blowing-up sequence of solutions of general conformally invariant fully nonlinear elliptic equations on Euclidean domains. We prove that (i) the distance between blow-up points is bounded from below by a universal positive number, (ii) the solutions are very close to a single standard bubble in a universal positive distance around each blow-up point, and (iii) the heights of these bubbles are comparable by a universal factor. As an application of this result, we establish a quantitative Liouville theorem.
\end{abstract}

Mathematics Subject Classification $35 \mathrm{~J} 60 \cdot 35 \mathrm{~B} 06 \cdot 35 \mathrm{~B} 53 \cdot 35 \mathrm{D} 40 \cdot 53 \mathrm{~A} 30 \cdot 53 \mathrm{C} 21$

\section{Contents}

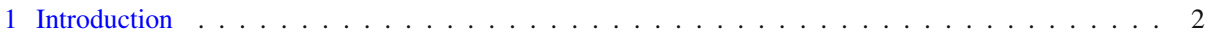

2 Non-quantitative Liouville theorems . . . . . . . . . . . . . . . . . . . . . 9

2.1 A gradient estimate . . . . . . . . . . . . . . . . . . . . . . . . . . . 9

2.2 Proof of Theorem $1.1 \ldots \ldots \ldots \ldots \ldots \ldots$

2.3 Proof of Theorem $1.2 \ldots \ldots \ldots \ldots \ldots \ldots \ldots \ldots$

3 Local gradient estimates . . . . . . . . . . . . . . . . . . . . . . . . . . . . . 19

4 Fine blow-up analysis . . . . . . . . . . . . . . . . . . . . . . . . 21

4.1 A quantitative centered Liouville-type result . . . . . . . . . . . . . . . . . . . . . . 21

Communicated by H. Brezis.

YanYan Li is partially supported by NSF Grant DMS-1501004.

YanYan Li

yyli@math.rutgers.edu

Luc Nguyen

luc.nguyen@maths.ox.ac.uk

1 Department of Mathematics, Rutgers University, 110 Frelinghuysen Road, Piscataway, NJ 08854, USA

2 Mathematical Institute and St Edmund Hall, University of Oxford, Andrew Wiles Building, Radcliffe Observatory Quarter, Woodstock Road, Oxford OX2 6GG, UK 
4.2 Detailed blow-up landscape . . . . . . . . . . . . . . . . . . . . . . . . . 27

5 A quantitative Liouville theorem . . . . . . . . . . . . . . . . . . . . . . . . . . . . . . . . . . 29

Appendix A: A remark on positive superharmonic functions . . . . . . . . . . . . . . . 30 Appendix B: A remark on viscosity solutions . . . . . . . . . . . . . . . . . . . . . . . 32 Appendix C: A calculus lemma . . . . . . . . . . . . . . . . . . . . . . . . . . 34

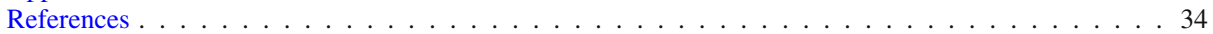

\section{Introduction}

The main goal of this paper is to give a fine analysis of blow-up solutions of conformally invariant fully nonlinear second order elliptic equations.

Let $n \geq 3$ be an integer and

$$
\Gamma \subset \mathbb{R}^{n} \text { be an open convex symmetric cone with vertex at the origin }
$$

satisfying

$$
\Gamma_{n} \subset \Gamma \subset \Gamma_{1},
$$

where

$$
\Gamma_{n}:=\left\{\lambda \in \mathbb{R}^{n} \mid \lambda_{i}>0 \forall i\right\}, \quad \Gamma_{1}:=\left\{\lambda \in \mathbb{R}^{n} \mid \sum_{i=1}^{n} \lambda_{i}>0\right\} .
$$

We assume that

$$
\begin{aligned}
& f \in C^{1}(\Gamma) \cap C^{0}(\bar{\Gamma}) \text { is symmetric in } \lambda_{i}, \\
& f>0, \quad \frac{\partial f}{\partial \lambda_{i}}>0 \text { in } \Gamma \forall i, \quad f=0 \text { on } \partial \Gamma, \\
& f(\lambda)<1 \quad \forall \lambda \in \Gamma \text { satisfying } \sum_{i=1}^{n} \lambda_{i}<\delta .
\end{aligned}
$$

In (1) and (3), the symmetric property of $\Gamma$ and $f$ is understood in the sense that if $\lambda \in \Gamma$ and $\tilde{\lambda}$ is a permutation of $\lambda$, then $\tilde{\lambda} \in \Gamma$ and $f(\tilde{\lambda})=f(\lambda)$. Also, throughout the paper,

whenever we write $f(\lambda)$, we implicitly assume that $\lambda \in \bar{\Gamma}$.

When $\Gamma \neq \Gamma_{1},(5)$ is a consequence of (3) and (4) (cf. [13, Proposition B.1]). However, this does not have to be the case when $\Gamma=\Gamma_{1}$, for example when

$$
f(\lambda)=\left(\sum_{i=1}^{n} \lambda_{i}\right)^{\frac{n-1}{n+3}}\left(\sum_{i=1}^{n} \lambda_{i}^{2}\right)^{\frac{2}{n+3}}
$$

Illuminating examples of $(f, \Gamma)$ are $(f, \Gamma)=\left(\sigma_{k}^{\frac{1}{k}}, \Gamma_{k}\right)$ where $\sigma_{k}(\lambda)=\sum \lambda_{i_{1}} \ldots \lambda_{i_{k}}$ is the $k$-th elementary symmetric function and

$$
\begin{aligned}
\Gamma_{k} & =\text { the connected component of }\left\{\lambda \in \mathbb{R}^{n}: \sigma_{k}(\lambda)>0\right\} \text { containing } \Gamma_{n} \\
& =\left\{\lambda \in \mathbb{R}^{n}: \sigma_{l}(\lambda)>0 \text { for all } 1 \leq l \leq k\right\} .
\end{aligned}
$$

Besides (1)-(5), $\left(\sigma_{k}^{\frac{1}{k}}, \Gamma_{k}\right)$ enjoys other nice and helpful properties, such as concavity and homogeneity properties of $\sigma_{k}^{1 / k}$, Newton's inequalities, divergence and variational structures, etc., which we do not assume in this paper. In particular, we would like to note that no concavity or homogeneity assumption on $f$ is being made in the present paper. 
For a positive $C^{2}$ function $u$, let $A^{u}$ be the $n \times n$ matrix with entries

$$
\left(A^{u}\right)_{i j}=-\frac{2}{n-2} u^{-\frac{n+2}{n-2}} \nabla_{i} \nabla_{j} u+\frac{2 n}{(n-2)^{2}} u^{-\frac{2 n}{n-2}} \nabla_{i} u \nabla_{j} u-\frac{2}{(n-2)^{2}} u^{-\frac{2 n}{n-2}}|\nabla u|^{2} \delta_{i j} .
$$

This is sometimes referred to as the conformal Hessian of $u$.

The conformal Hessian $A^{u}$ arises naturally in conformal geometry as follows. Recall that the Riemann curvature Riem $g$ of a Riemannian metric $g$ can be decomposed into traced and traceless parts as

$$
\text { Riem }_{g}=A_{g} \otimes g+W_{g},
$$

where $A_{g}=\frac{1}{n-2}\left(\operatorname{Ric}_{g}-\frac{1}{2(n-1)} R_{g} g\right), \operatorname{Ric}_{g}, R_{g}$ and $W_{g}$ are the Schouten curvature, the Ricci curvature, the scalar curvature and the Weyl curvature of $g$ and $\otimes$ denotes the Kulkarni-Nomizu product. While the (1,3)-valent Weyl curvature remains unchanged under a conformal change of the metric, the Schouten curvature is adjusted by a second order operator of the conformal factor. In particular, if we consider the metric $g_{u}:=u^{\frac{4}{n-2}} g_{\text {flat }}$ conformal to the flat metric $g_{\text {flat }}$ on $\mathbb{R}^{n}$, then the Schouten curvature $A_{g_{u}}$ of $g_{u}$ is given by the conformal Hessian in the form

$$
A_{g_{u}}=u^{\frac{4}{n-2}}\left(A^{u}\right)_{i j} d x^{i} d x^{j}
$$

Consequently, we have

$$
\lambda\left(A_{g_{u}}\right)=\lambda\left(A^{u}\right)
$$

where $\lambda\left(A_{g_{u}}\right)$ denotes the eigenvalues of $A_{g_{u}}$ with respect to the metric $g_{u}$ and $\lambda\left(A^{u}\right)$ denotes those of the matrix $A^{u}$.

$A^{u}$ enjoys a conformal invariance property, inherited from the conformal structure of $\mathbb{R}^{n}$, which will be of special importance in our treatment. Recall that a map $\varphi: \mathbb{R}^{n} \cup\{\infty\} \rightarrow$ $\mathbb{R}^{n} \cup\{\infty\}$ is called a Möbius transformation if it is the composition of finitely many of the following types of transformations:

- a translation: $x \mapsto x+\bar{x}$ where $\bar{x}$ is a given vector in $\mathbb{R}^{n}$,

- a dilation: $x \mapsto a x$ where $a$ is a given positive scalar,

- a Kelvin transformation: $x \mapsto \frac{x}{|x|^{2}}$.

For a function $u$ and a Möbius transformation $\varphi$, let

$$
u_{\varphi}=\left|J_{\varphi}\right|^{\frac{n-2}{2 n}} u \circ \varphi,
$$

where $J_{\varphi}$ is the Jacobian of $\varphi$. A calculation gives

$$
A^{u_{\varphi}}(x)=O_{\varphi}(x)^{t} A^{u}(\varphi(x)) O_{\varphi}(x)
$$

for some orthogonal $n \times n$ matrix $O_{\varphi}(x)$. In particular,

$$
\lambda\left(A^{u_{\varphi}}(x)\right)=\lambda\left(A^{u}(\varphi(x))\right) .
$$

The main result of this paper concerns an analysis on the behavior of a sequence $\left\{u_{k}\right\} \in$ $C^{2}\left(B_{3}(0)\right)$ satisfying

$$
f\left(\lambda\left(A^{u_{k}}\right)\right)=1, u_{k}>0, \text { in } B_{3}(0),
$$

and

$$
\sup _{B_{1}(0)} u_{k} \rightarrow \infty,
$$

where $(f, \Gamma)$ satisfies (1)-(5). Note that no other assumptions on $u_{k}$ is made. 
As is known, Eq. (8) is a fully nonlinear elliptic equation. Fully nonlinear elliptic equations involving $f\left(\lambda\left(\nabla^{2} u\right)\right)$ were investigated in the classic paper of Caffarelli et al. [2].

Our paper appears to be the first fine blow-up analysis in this fully nonlinear context. We expect this to serve as a crucial step in the study of the problem on Riemannian manifolds.

To obtain our result on fine analysis of blow-up solutions, we make use of the following Liouville theorems.

Theorem A ([8]) Let $(f, \Gamma)$ satisfy (1)-(4) and let $0<v \in C^{2}\left(\mathbb{R}^{n}\right)$ satisfy

$$
f\left(\lambda\left(A^{v}\right)\right)=1 \quad \text { in } \mathbb{R}^{n} .
$$

Then

$$
v(x) \equiv\left(\frac{a}{1+b^{2}|x-\bar{x}|^{2}}\right)^{\frac{n-2}{2}}, \quad x \in \mathbb{R}^{n}
$$

for some $\bar{x} \in \mathbb{R}^{n}$ and some positive constants $a$ and $b$ satisfying

$$
f\left(2 b^{2} a^{-2}, \ldots, 2 b^{2} a^{-2}\right)=1 .
$$

Theorem B ([12]) Let $\Gamma$ satisfy (1) and (2), and let $0<v \in C_{l o c}^{0,1}\left(\mathbb{R}^{n} \backslash\{0\}\right)$ satisfy

$$
\lambda\left(A^{v}\right) \in \partial \Gamma \text { on } \mathbb{R}^{n} \backslash\{0\}
$$

in the viscosity sense (see Definition 1.2 below). Then $v$ is radially symmetric about the origin and $v(r)$ is non-increasing in $r$.

For $(f, \Gamma)=\left(\frac{1}{2 n} \sigma_{1}, \Gamma_{1}\right)$, Eq. (8) is the critical exponent equation $-\Delta u=n(n-$ 2) $u^{(n+2) /(n-2)}$ and Theorem A was proved by Caffarelli et al. [1]. See also Gidas et al. [5] under some decay assumption of $u$ at infinity. For $(f, \Gamma)=\left(\sigma_{2}^{1 / 2}, \Gamma_{2}\right)$ in $\mathbb{R}^{4}$ and $v \in C_{l o c}^{1,1}\left(\mathbb{R}^{4}\right)$, the result was proved by Chang et al. [3].

In fact we need a stronger version of Theorem A (see Theorem 1.1) and a variant of Theorem B (see Theorem 1.2). For simplicity, readers are advised that in the main body of the paper

all theorems, propositions and lemmas hold under (1)-(5),

instead of the stated weaker hypotheses on $(f, \Gamma)$.

Theorem 1.1 Let $(f, \Gamma)$ satisfy

$\Gamma \subset \mathbb{R}^{n}$ be an open symmetric set,

$\Gamma \subset \Gamma_{1}$ and $\Gamma \cap\{\lambda+t \mu: t>0\}$ is convex for all $\lambda \in \Gamma, \mu \in \Gamma_{n}$,

$f \in C^{1}(\Gamma)$ is symmetric in $\lambda_{i}$ and $\frac{\partial f}{\partial \lambda_{i}}>0$ in $\Gamma \forall i$.

Assume that $0<v \in C^{0}\left(\mathbb{R}^{n}\right), 0<v_{k} \in C^{2}\left(B_{R_{k}}(0)\right), R_{k} \rightarrow \infty$,

$$
f\left(\lambda\left(A^{v_{k}}\right)\right)=1 \quad \text { in } B_{R_{k}}(0),
$$

and

$$
v_{k} \rightarrow \text { vin } C_{l o c}^{0}\left(\mathbb{R}^{n}\right) .
$$

Then either $v$ is constant or $v$ is of the form (11) for some $\bar{x} \in \mathbb{R}^{n}$ and some positive constants $a$ and $b$. 
If it holds in addition that

$$
\text { there exists } t_{0}>0 \text { such that } f\left(t_{0}, \ldots, t_{0}\right)<1 \text {, }
$$

then $v$ cannot be constant. If it holds further that

$$
\Gamma+\Gamma_{n}=\left\{\lambda+\mu: \lambda \in \Gamma, \mu \in \Gamma_{n}\right\} \subset \Gamma,
$$

then the constants $a$ and $b$ in (11) satisfy $\left(2 b^{2} a^{-2}, \ldots, 2 b^{2} a^{-2}\right) \in \Gamma$ and

$$
f\left(2 b^{2} a^{-2}, \ldots, 2 b^{2} a^{-2}\right)=1 .
$$

Remark 1.1 In Theorem 1.1, if condition (18) is dropped, the case that $v$ is constant can occur. See the counterexample in Remark 2.1.

Theorem 1.2 Let $(f, \Gamma)$ satisfy (13)-(15). Assume that $v_{*} \in C^{0}\left(\mathbb{R}^{n} \backslash\{0\}\right), 0<v_{k} \in$ $C^{2}\left(B_{R_{k}}(0) \backslash\{0\}\right), R_{k} \rightarrow \infty$,

$$
f\left(\lambda\left(A^{v_{k}}\right)\right)=1 \quad \text { in } B_{R_{k}}(0) \backslash\{0\},
$$

and, for some $M_{k} \rightarrow \infty$,

$$
M_{k} v_{k} \rightarrow v_{*} \text { in } C_{l o c}^{0}\left(\mathbb{R}^{n} \backslash\{0\}\right) .
$$

Then $v_{*}$ is radially symmetric about the origin, i.e. $v_{*}(x)=v_{*}(|x|)$. In particular, if $v_{k} \in$ $C^{2}\left(B_{R_{k}}(0)\right), f\left(\lambda\left(A^{v_{k}}\right)\right)=1$ in $B_{R_{k}}(0)$ and $M_{k} v_{k}$ converges to $v_{*}$ in $C_{\text {loc }}^{0}\left(\mathbb{R}^{n}\right)$, then $v_{*}$ is constant.

Remark 1.2 When $(f, \Gamma)$ satisfies (1)-(4) and an additional hypothesis that $f$ is homogeneous of positive degree, the function $v_{*}$ in Theorem 1.2 is a viscosity solution of (12) and the conclusion follows from Theorem $\mathrm{B}$. However, when $f$ is not homogeneous, $v_{*}$ is not necessarily a viscosity solution of (12).

It is not difficult to see that, under (1)-(4), the function $v$ in Theorem 1.1 is a viscosity solution of (10) (see Remark B.2). We have the following conjecture.

Conjecture Let $(f, \Gamma)$ satisfy (1)-(4), and let $0<v \in C_{l o c}^{0}\left(\mathbb{R}^{n}\right)$ be a viscosity solution of (10). Then $v$ is of the form (11) for some $\bar{x} \in \mathbb{R}^{n}$ and some positive constants $a$ and $b$.

The notion of viscosity solutions given below is consistent with that in [12].

Definition 1.1 A positive continuous function $v$ in an open set $\Omega \subset \mathbb{R}^{n}$ is a viscosity supersolution (respectively, subsolution) of

$$
f\left(\lambda\left(A^{v}\right)\right)=1, \text { in } \Omega,
$$

when the following holds: if $x_{0} \in \Omega, \varphi \in C^{2}(\Omega),(v-\varphi)\left(x_{0}\right)=0$, and $v-\varphi \geq 0$ near $x_{0}$, then

$$
f\left(\lambda\left(A^{\varphi}\left(x_{0}\right)\right) \geq 1\right.
$$

(respectively, if $(v-\varphi)\left(x_{0}\right)=0$, and $v-\varphi \leq 0$ near $x_{0}$, then either $\lambda\left(A^{\varphi}\left(x_{0}\right)\right) \in \mathbb{R}^{n} \backslash \bar{\Gamma}$ or $\left.f\left(\lambda\left(A^{\varphi}\left(x_{0}\right)\right)\right) \leq 1\right)$. We say that $v$ is a viscosity solution if it is both a viscosity supersolution and a viscosity subsolution. 
Definition 1.2 A positive continuous function $v$ in an open set $\Omega \subset \mathbb{R}^{n}$ is a viscosity supersolution (respectively, subsolution) of

$$
\lambda\left(A^{v}\right) \in \partial \Gamma, \text { in } \Omega,
$$

when the following holds: if $x_{0} \in \Omega, \varphi \in C^{2}(\Omega),(v-\varphi)\left(x_{0}\right)=0$, and $v-\varphi \geq 0$ near $x_{0}$, then

$$
\lambda\left(A^{\varphi}\left(x_{0}\right) \in \bar{\Gamma}\right.
$$

(respectively, if $(v-\varphi)\left(x_{0}\right)=0$, and $v-\varphi \leq 0$ near $x_{0}$, then either $\left.\lambda\left(A^{\varphi}\left(x_{0}\right)\right) \in \mathbb{R}^{n} \backslash \bar{\Gamma}\right)$. We say that $v$ is a viscosity solution if it is both a viscosity supersolution and a viscosity subsolution.

It is clear that for $C^{2}$ functions the notions of viscosity solutions and classical solutions coincide. Also, viscosity super- and sub-solutions are stable under uniform convergence, see "Appendix B".

Note that for any $\lambda=\left(\lambda_{1}, \ldots, \lambda_{n}\right) \in \Gamma, t_{1}=\max \lambda_{i}+1>0$ and $\left(t_{1}, \ldots, t_{1}\right) \in \lambda+\Gamma_{n}$. In other words, the sets $\lambda+\Gamma_{n}$ have non-empty intersection with the ray $\{(t, \ldots, t): t>0\}$. Thus, if $f^{-1}(1) \neq \emptyset$, then, in view of (4), there exists some $c>0$ such that $f(c, \ldots, c)=1$. In such situation, working with $\tilde{f}(\lambda):=f\left(\frac{c}{2} \lambda\right)$ instead of $f$, we may assume without loss of generality the following normalization condition

$$
f(2, \ldots, 2)=1 .
$$

Let

$$
U(x):=\left(\frac{1}{1+|x|^{2}}\right)^{\frac{n-2}{2}}, \quad x \in \mathbb{R}^{n} .
$$

A calculation gives

$$
A^{U} \equiv 2 I \text {. }
$$

With the normalization (21), $U$ satisfies

$$
f\left(\lambda\left(A^{U}\right)\right)=1 \text { on } \mathbb{R}^{n} .
$$

For $\bar{x} \in \mathbb{R}^{n}$ and $\mu>0$, let

$$
U^{\bar{x}, \mu}(x)=\mu U\left(\mu^{\frac{2}{n-2}}(x-\bar{x})\right)=\left(\frac{\mu^{\frac{2}{n-2}}}{1+\mu^{\frac{4}{n-2}}|x-\bar{x}|^{2}}\right)^{\frac{n-2}{2}} .
$$

Note that, in the sense of (6), $U^{\bar{x}, \mu}=U_{\varphi}$ with $\varphi(x)=\mu^{\frac{2}{n-2}}(x-\bar{x})$. Hence, by the conformal invariance (7), for any $\bar{x} \in \mathbb{R}^{n}$ and $\mu>0$,

$$
f\left(\lambda\left(A^{U_{\bar{x}, \mu}}\right)\right)=1 \text { on } \mathbb{R}^{n} .
$$

Theorem 1.3 Let $(f, \Gamma)$ satisfy (13)-(15), (18)-(19), (5) and the normalization condition (21). Let $\epsilon \in(0,1 / 2]$. There exist constants $\bar{m}=\bar{m}(f, \Gamma) \geq 1, K=K(f, \Gamma)>1$, $\delta_{*}=\delta_{*}(\epsilon, f, \Gamma)>0, C_{*}=C_{*}(\epsilon, f, \Gamma)>1$ such that for any positive $u \in C^{2}\left(B_{3}(0)\right)$ satisfying ${ }^{1}$

$$
f\left(\lambda\left(A^{u}\right)\right)=1 \text { in } B_{3}(0) \text { and } \sup _{B_{1}(0)} u \geq C_{*},
$$

1 Note that, $\bar{m}$ and $K$ are independent of $\epsilon$. 
there exists $\left\{x^{1}, \ldots, x^{m}\right\} \subset B_{2}(0)$ with $1 \leq m \leq \bar{m}$ satisfying

(i) $u\left(x^{1}\right) \geq \sup _{B_{1}(0)} u$,

(ii) $\left|x^{i}-x^{j}\right| \geq \frac{1}{K}$ for all $1 \leq i \neq j \leq m$,

(iii) $\frac{1}{K} \leq \frac{u\left(x^{i}\right)}{u\left(x^{j}\right)} \leq K$ for all $1 \leq i, j \leq m$,

(iv) $\left|u(x)-U^{x^{i}, u\left(x^{i}\right)}(x)\right| \leq \epsilon U^{x^{i}, u\left(x^{i}\right)}(x)$ for all $1 \leq i \leq m, x \in B_{\delta_{*}}\left(x^{i}\right)$,

(v) $\frac{1}{K \delta_{*}^{n-2} u\left(x^{1}\right)} \leq u(x) \leq \frac{K}{\delta_{*}^{n-2} u\left(x^{1}\right)}$ for all $x \in B_{\frac{3}{2}}(0) \backslash \cup_{i=1}^{m} B_{\delta_{*}}\left(x^{i}\right)$,

(vi) $u\left(x^{i}\right)=\sup _{B_{\delta_{*}}\left(x^{i}\right)} u$.

Remark 1.3 If it holds further that $\sup _{B_{1}(0)} u>\frac{K^{1 / 2}}{\delta_{*} \frac{n-2}{2}}$, then

$$
\min _{1 \leq i \leq m} \operatorname{dist}\left(x^{i}, B_{1}(0)\right) \leq\left[\sup _{B_{1}(0)} u\right]^{-\frac{2}{n-2}} .
$$

To see this, let $x_{*}$ be a point in $\bar{B}_{1}(0)$ such that $u\left(x_{*}\right)=\sup _{B_{1}(0)} u$. In view of (v) and the stated condition on $\sup _{B_{1}(0)} u, x_{*}$ belongs to some ball $B_{\delta_{*}}\left(x^{i_{0}}\right)$. By (iv), we then have

$$
u\left(x_{*}\right) \leq 2 U^{x^{i_{0}}, u\left(x^{i_{0}}\right)}\left(x_{*}\right) \leq \frac{1}{\left|x_{*}-x^{i_{0}}\right|^{\frac{n-2}{2}}},
$$

which implies the assertion.

Theorem 1.3 can be stated equivalently as follows.

Theorem 1.4 Let $(f, \Gamma)$ satisfy (13)-(15), (18)-(19), (5) and the normalization condition (21). Assume that $0<u_{k} \in C^{2}\left(B_{3}(0)\right)$ satisfy (8) and (9). Let $\epsilon \in(0,1 / 2]$. Then there exist $\bar{m}=\bar{m}(f, \Gamma) \geq 1, K(f, \Gamma)>1$ and $\delta_{*}=\delta_{*}(\epsilon, f, \Gamma)>0$ such that, after passing to a subsequence, still denoted by $u_{k}$, there exists $\left\{x_{k}^{1}, \ldots, x_{k}^{m}\right\} \subset B_{2}(0)(1 \leq m \leq \bar{m})$ satisfying ${ }^{2}$

(i) $u_{k}\left(x_{k}^{1}\right) \geq \sup _{B_{1}(0)} u_{k}$,

(ii) $\left|x_{k}^{i}-x_{k}^{j}\right| \geq \frac{1}{K}$ for all $k \geq 1,1 \leq i \neq j \leq m$,

(iii) $\frac{1}{K} \leq \frac{u_{k}\left(x_{k}^{i}\right)}{u_{k}\left(x_{k}^{j}\right)} \leq K$ for all $k \geq 1,1 \leq i, j \leq m$,

(iv) $\left|u_{k}(x)-U^{x_{k}^{i}, u_{k}\left(x_{k}^{i}\right)}(x)\right| \leq \epsilon U^{x_{k}^{i}, u_{k}\left(x_{k}^{i}\right)}(x)$ for all $k \geq 1,1 \leq i \leq m, x \in B_{\delta_{*}}\left(x_{k}^{i}\right)$,

(v) $\frac{1}{K \delta_{*}^{n-2} u_{k}\left(x_{k}^{1}\right)} \leq u_{k}(x) \leq \frac{K}{\delta_{*}^{n-2} u_{k}\left(x_{k}^{1}\right)}$ for all $k \geq 1, x \in B_{\frac{3}{2}}(0) \backslash \cup_{i=1}^{m} B_{\delta_{*}}\left(x_{k}^{i}\right)$,

(vi) $u_{k}\left(x_{k}^{i}\right)=\sup _{B_{\delta_{*}}\left(x_{k}^{i}\right)} u_{k}$.

Remark 1.4 By Remark 1.3, we have

$$
\operatorname{dist}\left(\left\{x_{k}^{1}, \ldots, x_{k}^{m}\right\}, B_{1}(0)\right) \rightarrow 0 \text { as } k \rightarrow \infty .
$$

When $(f, \Gamma)=\left(\frac{1}{2 n} \sigma_{1}, \Gamma_{1}\right)$, Eq. (8) is $-\Delta u_{k}=n(n-2) u_{k}^{\frac{n+2}{n-2}}$ and Theorem 1.4 in this case was proved by Schoen [16].

See Li [9] and Chen and Lin [4] for analogous results for the equation $-\Delta u_{k}=K(x) u_{k}^{\frac{n+2}{n-2}}$. In Theorems 1.3 and $1.4, B_{1}(0), B_{2}(0)$ and $B_{3}(0)$ can be replaced respectively by $B_{r_{1}}(0)$, $B_{r_{2}}(0)$ and $B_{r_{3}}(0), 0<r_{1}<r_{2}<r_{3}$, and in this case the constants $\bar{m}, K, \delta_{*}$ and $C_{*}$ depend also on $r_{1}, r_{2}$ and $r_{3}$.

The following is a quantitative version of Theorem A, and is related to Theorems 1.1 and 1.3.

2 The constant $m$ is independent of $k$. 
Theorem 1.5 (Quantitative Liouville Theorem) Let ( $f, \Gamma$ ) satisfy (13)-(15), (18)-(19), (5) and the normalization condition (21), and let $\gamma, r_{1}>0$ be constants. Then, for every $\epsilon \epsilon$ $(0,1 / 2]$, there exist some constants $\delta_{*}>0, R^{*}>0$, depending only on $(f, \Gamma), \gamma, r_{1}$ and $\epsilon$, such that if $0<v \in C^{2}\left(B_{R}(0)\right)$ for some $R \geq R^{*}$,

$$
f\left(\lambda\left(A^{v}\right)=1 \text { in } B_{R}(0),\right.
$$

and

$$
v \geq \gamma \quad \text { in } B_{r_{1}}(0)
$$

then, for some $\bar{x} \in \mathbb{R}^{n}$ satisfying

$$
v(\bar{x})=\max _{B_{\delta * R}(0)} v \leq \frac{2^{n-1}}{\gamma r_{1}^{n-2}}, \quad|\bar{x}| \leq 2^{\frac{1}{n-2}} \gamma^{-\frac{2}{n-2}},
$$

there holds

$$
\left|v(y)-U^{\bar{x}, v(\bar{x})}(y)\right| \leq \epsilon U^{\bar{x}, v(\bar{x})}(y), \quad \forall|y-\bar{x}| \leq \delta_{*} R .
$$

Remark 1.5 The constant $\delta_{*}$ in Theorems 1.3 and 1.5 can be chosen the same.

Remark 1.6 An analogous result for the degenerate elliptic equation $\lambda\left(A^{v}\right) \in \partial \Gamma$ is a consequence of the local gradient estimate [13, Theorem 1.5].

An ingredient in our proof of Theorems 1.3 and 1.4 is the following local gradient estimate, which follows from Theorem 1.2 and the proof of [12, Theorem 1.10].

Theorem 1.6 Let $(f, \Gamma)$ satisfy (13)-(15) and let $v \in C^{2}\left(B_{2}(0)\right)$ satisfy, for some constant $b>0$,

$$
0<v \leq b \quad \text { in } B_{2}(0) \text {, }
$$

and

$$
f\left(\lambda\left(A^{v}\right)\right)=1 \quad \text { in } B_{2}(0) .
$$

Then, for some constant $C$ depending only on $(f, \Gamma)$ and $b$,

$$
|\nabla \ln v| \leq C \text { in } B_{1}(0) .
$$

For $(f, \Gamma)=\left(\sigma_{k}^{1 / k}, \Gamma_{k}\right)$, the result was proved by Guan and Wang [6].

When $(f, \Gamma)$ satisfies (1)-(4) and is homogeneous of positive degree, Theorem 1.6 was proved in [12].

The rest of the paper is organized as follows. We start in Sect. 2 with the proof of Theorems 1.1 and 1.2. We then prove Theorem 1.6 in Sect. 3. In Sect. 4, we first establish an intermediate quantitative Liouville result and then use it to prove Theorem 1.3. In Sect. 5, we prove Theorem 1.5 as an application of Theorem 1.3. In "Appendix A", we present a lemma about super-harmonic functions which is used in the body of the paper. In "Appendix B", we include a relevant remark on the limit of viscosity solutions of elliptic PDE. Finally we collect in "Appendix C" some relevant calculus lemmas. 


\section{Non-quantitative Liouville theorems}

In this section, we prove Theorems 1.1 and 1.2. We use the method of moving spheres and establish along the way, as a tool, a gradient estimate which is in a sense weaker than that in Theorem 1.6 but suffices for the moment. (Note that the proof of Theorem 1.6 relies on Theorem 1.2.)

\subsection{A gradient estimate}

Theorem 2.1 Let $(f, \Gamma)$ satisfy (13), (15) and

$$
\Gamma \cap\{\lambda+t \mu: t>0\} \text { is convex for all } \lambda \in \Gamma, \mu \in \Gamma_{n} .
$$

Let $0<v \in C^{2}\left(B_{2}(0)\right)$ satisfy, for some constant $\theta>1$,

$$
\sup _{B_{2}(0)} v \leq \theta \inf _{B_{2}(0)} v
$$

and

$$
f\left(\lambda\left(A^{v}\right)\right)=1 \quad \text { in } B_{2}(0) .
$$

Then, for some constant $C$ depending only on $n$ and $\theta$,

$$
|\nabla \ln v| \leq C \text { in } B_{1}(0) .
$$

This type of gradient estimate was established and used in various work of the first named author and his collaborators under less general hypothesis on $(f, \Gamma)$. It turns out that the same proof works in the current situation. We give a detailed sketch here for completeness.

We use the method of moving spheres as in $[7,8,14,15]$. For a function $w$ defined on a subset of $\mathbb{R}^{n}$, we define

$$
w_{x, \lambda}(y)=\frac{\lambda^{n-2}}{|y-x|^{n-2}} w\left(x+\frac{\lambda^{2}(y-x)}{|y-x|^{2}}\right)
$$

wherever the expression makes sense. We will use $w_{\lambda}$ to denote $w_{0, \lambda}$. We start with a simple result.

Lemma 2.1 Let $R>0$ and $w$ be a positive Lipschitz function in $\bar{B}_{R}(0)$ such that, for some $L>0$,

$$
|\ln w(y)-\ln w(z)| \leq L|y-z| \text { for all } y, z \in \bar{B}_{R}(0) .
$$

Then for $\underline{\lambda}=\min \left(\frac{n-2}{2 L}, \frac{R}{2}\right)$ we have

$$
w_{\lambda} \leq w \text { in } B_{\underline{\lambda}}(0) \backslash B_{\lambda}, \forall 0<\lambda<\underline{\lambda} .
$$

Proof Write $w$ in polar coordinates $w(r, \theta)$. It is easy to see that (30) is equivalent to

$$
\left.r^{\frac{n-2}{2}} w(r, \theta)\right) \leq s^{\frac{n-2}{2}} w(s, \theta), \quad \forall 0<r<s<\underline{\lambda}, \forall \theta .
$$

Estimate (31) is readily seen from the estimates

$$
\begin{aligned}
\ln w(s, \theta)-\ln w(r, \theta) & \geq-L|s-r| \geq-\frac{n-2}{2 \underline{\lambda}}(s-r) \\
& \geq-\frac{n-2}{2}[\ln s-\ln r] .
\end{aligned}
$$

Lemma 2.1 is established. 
Proof of Theorem 2.1 By Lemma 2.1, there exists some $r_{0} \in(0,1 / 3)$ such that

$$
v_{x, \lambda} \leq v \text { in } B_{r_{0}}(x) \backslash B_{\lambda}(x) \text { for all } \lambda \in\left(0, r_{0}\right) \text { and } x \in B_{4 / 3}(0) .
$$

It is easy to see that, for some $r_{1} \in\left(0, r_{0}\right)$,

$$
v_{x, \lambda} \leq v \text { in } B_{5 / 3}(0) \backslash B_{r_{0}}(x) \text { for all } \lambda \in\left(0, r_{1}\right) \text { and } x \in B_{4 / 3}(0) .
$$

We then define, for $x \in B_{4 / 3}(0)$,

$$
\bar{\lambda}(x)=\sup \left\{\lambda \in(0,5 / 3-|x|): v_{x, \lambda} \leq v \text { in } B_{5 / 3}(0) \backslash B_{\lambda}(x)\right\} .
$$

We have

$$
v_{x, \bar{\lambda}(x)} \leq v \text { in } B_{5 / 3}(0) \backslash B_{\bar{\lambda}(x)}(x),
$$

By the conformal invariance $(7), v_{x, \bar{\lambda}(x)}$ satisfies

$$
f\left(\lambda\left(A^{\left.v_{x, \bar{\lambda}(x)}\right)}\right)=1, \quad \text { in } B_{5 / 3}(0) \backslash \overline{B_{\bar{\lambda}(x)}(x)} .\right.
$$

Using the above two displayed equations, the definition of $\bar{\lambda}(x)$, and using the ellipticity of the equation satisfied by $v$ and $v_{x, \bar{\lambda}(x)}$, we can apply the strong maximum principle and Hopf Lemma to infer that either $\bar{\lambda}(x)=5 / 3-|x|$ or there exists some $y \in \partial B_{5 / 3}(0)$ such that

$$
v_{x, \bar{\lambda}(x)}(y)=v(y)
$$

- see the proof of $[8$, Lemma 4.5].

In the latter case, (29) implies that

$$
\theta \geq \frac{v\left(x+\frac{\bar{\lambda}(x)^{2}(y-x)}{|y-x|^{2}}\right)}{v(y)}=\frac{|y-x|^{n-2}}{\bar{\lambda}(x)^{n-2}} \geq \frac{(5 / 3-|x|)^{n-2}}{\bar{\lambda}(x)^{n-2}} .
$$

In either case, we obtain that

$$
\bar{\lambda}(x) \geq c(n, \theta)>0 \text { for all } x \in B_{4 / 3}(0) .
$$

The conclusion then follows from [8, Lemma A.2].

\subsection{Proof of Theorem 1.1}

Remark 2.1 If we drop condition (18), the case that $v$ is constant in Theorem 1.1 can occur. For example, consider $n \geq 3$ and

$$
\begin{aligned}
& f(\lambda)=\sigma_{2}(\lambda)+1, \quad \Gamma=\left\{\lambda \in \mathbb{R}^{n} \mid \sum_{j=1}^{n} \lambda_{j}-\lambda_{i}>0 \text { for all } i=1, \ldots, n\right\}, \\
& v_{k}(x)=\left(\frac{1}{R_{k}^{n+4}}\left|x-x_{k}\right|^{-\frac{n-4}{2}}+1\right)^{\frac{2(n-2)}{n-4}} \text { for }|x|<\frac{\left|x_{k}\right|}{2}=R_{k} \rightarrow \infty .
\end{aligned}
$$

It is readily seen that $f(t, \ldots, t)>1$ for all $(t, \ldots, t) \in \Gamma, v_{k}$ satisfies (16) (cf. [13, Theorem 1.6]) and $v_{k} \rightarrow 1$ in $C_{l o c}^{0}\left(\mathbb{R}^{n}\right)$.

Proof of Theorem 1.1 We may assume that $R_{k} \geq 5$ for all $k$.

Clearly, for every $\beta>1$, there exists some positive constant $C(\beta)$, independent of $k$, such that $1 / C(\beta) \leq v_{k} \leq C(\beta)$ in $B_{\beta}(0)$. It follows from Theorem 2.1 that $\left|\nabla \ln v_{k}\right| \leq C^{\prime}(\beta)$ in 
$B_{\beta / 2}(0)$. It follows, after passing to a subsequence, that for every $0<\alpha<1, v_{k} \rightarrow v$ in $C_{l o c}^{\alpha}\left(\mathbb{R}^{n}\right), v \in C_{\text {loc }}^{0,1}\left(\mathbb{R}^{n}\right)$ and $v$ is super-harmonic on $\mathbb{R}^{n}$.

Using the positivity, the superharmonic of $v$, and the maximum principle, we can find $c_{0}>0$ such that

$$
v(y) \geq 2 c_{0}|y|^{2-n}, \quad \forall|y| \geq 1 .
$$

Passing to a subsequence and shrinking $R_{k}$ and $c_{0}>0$, if necessary, we may assume that

$$
\left|v_{k}(y)-v(y)\right| \leq R_{k}^{-n}, \quad \forall|y| \leq R_{k},
$$

and

$$
v_{k}(y) \geq c_{0}(1+|y|)^{2-n}, \quad \forall|y|<R_{k} .
$$

Lemma 2.2 Under the hypotheses of Theorem 1.1, there exists a function $\lambda^{(0)}: \mathbb{R}^{n} \rightarrow$ $(0, \infty)$ such that, for all $k$,

$$
\left(v_{k}\right)_{x, \lambda} \leq v_{k} \text { in } B_{R_{k}}(0) \backslash B_{\lambda}(x), \forall 0<\lambda<\lambda^{(0)}(x) \text { and }|x| \leq \frac{R_{k}}{5} .
$$

Proof For $|x| \leq \frac{R_{k}}{5}$, we have, by (33) and (34), for all $k$ that

$$
\frac{1}{c_{1}(x)} \leq v_{k} \leq c_{1}(x) \text { in } B_{4 r_{1}(x)}(x) \subset B_{R_{k}}(0),
$$

where

$$
r_{1}(x)=\frac{1}{4}+|x|, \text { and } c_{1}(x)=\max \left\{1+\sup _{B_{4 r_{1}(x)}(x)} v, \frac{1}{c_{0}}\left(1+|x|+4 r_{1}(x)\right)^{n-2}\right\} .
$$

By (35) and Theorem 2.1, there exists $c_{2}(x)>0$, independent of $k$, such that

$$
\left|\nabla \ln v_{k}\right| \leq c_{2}(x) \text { in } B_{2 r_{1}(x)}(x) .
$$

Thus, by Lemma 2.1, we can find $0<\lambda_{1}(x)<r_{1}(x)$ independent of $k$ such that

$$
\left(v_{k}\right)_{x, \lambda} \leq v_{k} \text { in } B_{\lambda_{1}(x)}(x) \backslash B_{\lambda}(x) \forall 0<\lambda<\lambda_{1}(x) .
$$

For $0<\lambda<\lambda_{1}(x)$, we have, using (35), that

$$
\left(v_{k}\right)_{x, \lambda}(y) \leq \frac{\lambda^{n-2} c_{1}(x)}{|y-x|^{n-2}}, \quad \forall y \in B_{R_{k}}(0) \backslash B_{\lambda}(x) .
$$

When $y \in B_{R_{k}}(0) \backslash B_{4 r_{1}(x)}(x), \frac{1}{2}(1+|y|)<|y-x|$ and we obtain, using (37) and (34), that

$$
\left(v_{k}\right)_{x, \lambda}(y) \leq \frac{(2 \lambda)^{n-2} c_{1}(x)}{(1+|y|)^{n-2}} \leq \frac{(2 \lambda)^{n-2} c_{1}(x)}{c_{0}} v_{k}(y) .
$$

When $y \in B_{4 r_{1}(x)}(x) \backslash B_{\lambda_{1}(x)}(x), 1+|y| \leq 2(1+3|x|),|y-x| \geq \lambda_{1}(x)$ and we obtain, using (37) and (34), that

$$
\left(v_{k}\right)_{x, \lambda}(y) \leq \frac{\lambda^{n-2} c_{1}(x)}{\lambda_{1}(x)^{n-2}} \leq \frac{(2 \lambda)^{n-2} c_{1}(x)(1+3|x|)^{n-2}}{\lambda_{1}(x)^{n-2} c_{0}} v_{k}(y) .
$$

Letting

$$
\lambda^{(0)}(x)=\min \left\{\lambda_{1}(x), \frac{\lambda_{1}(x)}{2(1+3|x|)}\left[\frac{c_{1}(x)}{c_{0}}\right]^{\frac{1}{n-2}}\right\} \leq \lambda_{1}(x),
$$


we derive from (38) and (39) that

$$
\left(v_{k}\right)_{x, \lambda} \leq v_{k} \text { in } B_{R_{k}}(0) \backslash B_{\lambda_{1}(x)}(x) \text { and } 0<\lambda<\lambda^{(0)}(x) .
$$

Lemma 2.2 follows from (36) and (40).

Define, for $x \in \mathbb{R}^{n}$ and $|x| \leq R_{k} / 5$, that

$$
\bar{\lambda}_{k}(x)=\sup \left\{0<\mu \leq \frac{R_{k}}{5} \mid\left(v_{k}\right)_{x, \lambda} \leq v_{k} \text { in } B_{R_{k}}(0) \backslash B_{\lambda}(x), \forall 0<\lambda<\mu\right\} .
$$

By Lemma 2.2,

$$
\bar{\lambda}(x):=\liminf _{k \rightarrow \infty} \bar{\lambda}_{k}(x) \in\left[\lambda^{(0)}(x), \infty\right], \quad x \in \mathbb{R}^{n} .
$$

By (32),

$$
\alpha:=\liminf _{|y| \rightarrow \infty}|y|^{n-2} v(y) \in(0, \infty] .
$$

Lemma 2.3 Assume (13)-(15). Then either $v$ is constant or

$$
\alpha=\lim _{|y| \rightarrow \infty}|y|^{n-2} v_{x, \bar{\lambda}(x)}(y)=\bar{\lambda}(x)^{n-2} v(x)<\infty, \quad \forall x \in \mathbb{R}^{n} .
$$

Proof Step 1. If $\bar{\lambda}(x)<\infty$ for some $x \in \mathbb{R}^{n}$, then

$$
\alpha=\lim _{|y| \rightarrow \infty}|y|^{n-2} v_{x, \bar{\lambda}(x)}(y)=\bar{\lambda}(x)^{n-2} v(x)<\infty .
$$

Since $\bar{\lambda}(x)<\infty$, we have, along a subsequence, $\bar{\lambda}_{k}(x) \rightarrow \bar{\lambda}(x)$-but for simplicity, we still use $\left\{\bar{\lambda}_{k}(x)\right\},\left\{v_{k}\right\}$, etc to denote the subsequence. By the definition of $\bar{\lambda}_{k}(x)$, we have

$$
\left(v_{k}\right)_{x, \bar{\lambda}_{k}(x)} \leq v_{k} \text { in } B_{R_{k}}(0) \backslash B_{\bar{\lambda}_{k}(x)}(x),
$$

By the conformal invariance $(7),\left(v_{k}\right)_{x, \bar{\lambda}_{k}(x)}$ satisfies

$$
f\left(\lambda \left(A^{\left.\left.\left(v_{k}\right)_{x, \bar{\lambda}_{k}(x)}\right)\right)}=1, \quad \text { in } B_{R_{k}}(0) \backslash \overline{B_{\bar{\lambda}_{k}(x)}(x)} .\right.\right.
$$

Using (16), (41), (42), the definition of $\bar{\lambda}_{k}(x)$, and using the ellipticity of the equation satisfied by $v_{k}$ and $\left(v_{k}\right)_{x, \bar{\lambda}_{k}(x)}$, we can apply the strong maximum principle and Hopf Lemma to infer the existence of some $y_{k} \in \partial B_{R_{k}}(0)$ such that

$$
\left(v_{k}\right)_{x, \bar{\lambda}_{k}(x)}\left(y_{k}\right)=v_{k}\left(y_{k}\right)
$$

— see the proof of [8, Lemma 4.5].

It follows that

$$
\lim _{k \rightarrow \infty}\left|y_{k}\right|^{n-2} v_{k}\left(y_{k}\right)=\lim _{k \rightarrow \infty}\left|y_{k}\right|^{n-2}\left(v_{k}\right)_{x, \bar{\lambda}_{k}(x)}\left(y_{k}\right)=(\bar{\lambda}(x))^{n-2} v(x) .
$$

This implies, in view of (33), that

$$
\alpha \leq \lim _{k \rightarrow \infty}\left|y_{k}\right|^{n-2} v\left(y_{k}\right)=\bar{\lambda}(x)^{n-2} v(x)=\lim _{|y| \rightarrow \infty}|y|^{n-2} v_{x, \bar{\lambda}(x)}(y)<\infty .
$$

On the other hand, if $\hat{y}_{i}$ is such that $\left|\hat{y}_{i}\right| \rightarrow \infty$ and

$$
\alpha=\lim _{i \rightarrow \infty}\left|y_{i}\right|^{n-2} v\left(y_{i}\right)
$$


then, since $v_{x, \bar{\lambda}(x)} \leq v$ in $\mathbb{R}^{n} \backslash B_{\bar{\lambda}(x)}(x)$, we have

$$
v\left(y_{i}\right) \geq \frac{\bar{\lambda}(x)^{n-2}}{\left|y_{i}-x\right|^{n-2}} v\left(x+\frac{\bar{\lambda}(x)^{2}\left(y_{i}-x\right)}{\left|y_{i}-x\right|^{2}}\right) .
$$

This gives

$$
\alpha=\lim _{i \rightarrow \infty}\left|y_{i}\right|^{n-2} v\left(y_{i}\right) \geq \bar{\lambda}(x)^{n-2} v(x) .
$$

Step 1 is established.

Step 2. It remains to show that either $v$ is constant or, for every $x \in \mathbb{R}^{n}, \bar{\lambda}(x)<\infty$.

To this end, we show that if $\bar{\lambda}(x)=\infty$ for some $x \in \mathbb{R}^{n}$, then $v$ is constant. Indeed, assume that $\bar{\lambda}_{k}(x) \rightarrow \infty$ as $k \rightarrow \infty$. We easily derive from this and the convergence of $v_{k}$ to $v$ that

$$
v_{x, \lambda} \leq v \text { in } \mathbb{R}^{n} \backslash B_{\lambda}(x) \forall \lambda>0 .
$$

The above is equivalent to the property that for every fixed unit vector $e, r^{\frac{n-2}{2}} v(x+r e)$ is non-decreasing in $r$. Thus

$$
r^{n-2} \min _{\partial B_{r}(x)} v \geq r^{\frac{n-2}{2}} \min _{\partial B_{1}(x)} v \forall r \geq 1 .
$$

In particular, $\alpha=\liminf _{|y| \rightarrow \infty}|y|^{n-2} v(y)=\infty$. This implies, by Step 1 , that $\bar{\lambda}(x)=\infty$ for every $x \in \mathbb{R}^{n}$, and therefore (43) holds for every $x \in \mathbb{R}^{n}$. This implies that $v$ is a constant, see Corollary C.1.

Lemma 2.4 Assume (13)-(15) and (18). Then the function $v$ in Theorem 1.1 cannot be constant.

Proof Fix some $t>0$ for the moment. Set $\varphi(x)=v(0)-t|x|^{2}$ and fix some $r>0$ such that $\varphi>0$ in $B_{r}(0)$ and $\varphi<v_{k}$ on $\partial B_{r}(0)$ for all sufficiently large $k$. Let

$$
\gamma_{k}=\sup _{B_{r}(0)}\left(\varphi-v_{k}\right) \text { and } \varphi_{k}=\varphi-\gamma_{k} .
$$

Then $\varphi_{k} \leq v_{k}$ in $B_{r}(0)$ and $\varphi_{k}\left(x_{k}\right)=v_{k}\left(x_{k}\right)$ for some $x_{k} \in \bar{B}_{r}(0)$. Noting that

$$
\gamma_{k}=(\varphi-v)\left(x_{k}\right)-\left(v_{k}-v\right)\left(x_{k}\right)=-t\left|x_{k}\right|^{2}-\left(v_{k}-v\right)\left(x_{k}\right)
$$

and

$$
\lim _{k \rightarrow \infty} \gamma_{k}=\sup _{B_{r}(0)}(\varphi-v)=0
$$

we deduce that $x_{k} \rightarrow 0$. This leads to

$$
\varphi_{k}\left(x_{k}\right)=v_{k}\left(x_{k}\right), \nabla \varphi_{k}\left(x_{k}\right)=\nabla v_{k}\left(x_{k}\right), \nabla^{2} \varphi_{k}\left(x_{k}\right) \leq \nabla^{2} v_{k}\left(x_{k}\right)
$$

and

$$
A^{\varphi_{k}}\left(x_{k}\right) \geq A^{v_{k}}\left(x_{k}\right) .
$$

Noting that there is some $C>0$ independent of $\delta$ and $k$ such that, for large $k$,

$$
A^{\varphi_{k}}\left(x_{k}\right) \leq\left(\frac{4}{n-2} v(0)^{-\frac{n+2}{n-2}} t+C \delta\right) I .
$$


Thus, we can select $t$ and $\delta$ such that

$$
A^{v_{k}}\left(x_{k}\right) \leq A^{\varphi_{k}}\left(x_{k}\right) \leq t_{0} I .
$$

where $t_{0}$ is the constant in (18). Since $f\left(\lambda\left(A^{v_{k}}\left(x_{k}\right)\right)\right)=1$, this contradicts (14), (15) and (18).

Recall that $0<v \in C_{l o c}^{0,1}\left(\mathbb{R}^{n}\right), \Delta v \leq 0$ in $\mathbb{R}^{n}$, and it remains to consider the case that, for every $x \in \mathbb{R}^{n}$, there exists $0<\bar{\lambda}(x)<\infty$ such that

$$
v_{x, \bar{\lambda}(x)}(y) \leq v(y), \forall|y-x| \geq \bar{\lambda}(x),
$$

and

$$
\lim _{|y| \rightarrow \infty}|y|^{n-2} v_{x, \bar{\lambda}(x)}(y)=\alpha:=\liminf _{|y| \rightarrow \infty}|y|^{n-2} v(y)<\infty .
$$

If $v$ is in $C^{2}\left(\mathbb{R}^{n}\right)$, the conclusion of Theorem 1.1 follows from the proof of Theorem 1.3 in [8]. An observation made in [11] easily allows the proof to hold for $v \in C_{l o c}^{0,1}\left(\mathbb{R}^{n}\right)$. For readers' convenience, we outline the proof below.

Let $\psi(y)=\frac{y}{|y|^{2}}$. We denote

$$
v_{\psi}:=\left|J_{\psi}\right|^{\frac{n-2}{2 n}} v \circ \psi
$$

We know that $\lambda\left(A^{v_{\psi}}(y)\right)=\lambda\left(A^{v}(\psi(y))\right.$. Namely, $A^{v_{\psi}}(y)$ and $A^{v}(\psi(y))$ differ only by an orthogonal conjugation.

Introduce

$$
w^{(x)}:=\left(v_{x, \bar{\lambda}(x)}\right) \psi, x \in \mathbb{R}^{n} .
$$

We deduce from the above properties of $v$ that for every $x \in \mathbb{R}^{n}$, there exists some $\delta(x)>0$ such that

$$
\begin{aligned}
& v_{\psi} \geq w^{(x)} \text { in } B_{\delta(x)}(0) \backslash\{0\}, \\
& w^{(x)}(0)=\alpha=\liminf _{y \rightarrow 0} v_{\psi}(y), \\
& \Delta v_{\psi} \leq 0, \text { in } B_{\delta(x)}(0) \backslash\{0\},
\end{aligned}
$$

Let $D=\left\{x \in \mathbb{R}^{n} \mid v\right.$ is differentiable at $\left.x\right\}$. Since $v \in C_{l o c}^{0,1}\left(\mathbb{R}^{n}\right)$, the Lebesgue measure of $\mathbb{R}^{n} \backslash D$ is 0 . It is clear that $w^{(x)}(y)$ is differentiable at $y=0$ if $v$ is differentiable at $x$.

By [8, Lemma 4.1],

$$
\nabla w^{(x)}(0)=\nabla w^{(\tilde{x})}(0), \quad \forall x, \tilde{x} \in D .
$$

Namely, for some $V \in \mathbb{R}^{n}$,

$$
\nabla w^{(x)}(0)=V, \forall x \in D
$$

A calculation yields

$$
\nabla w^{(x)}(0)=(n-2) \alpha x+\alpha^{\frac{n}{n-2}} v(x)^{\frac{n}{n-2}} \nabla v(x) .
$$

Thus

$$
\nabla_{x}\left(\frac{n-2}{2} \alpha^{\frac{n}{n-2}} v(x)^{-\frac{2}{n-2}}-\frac{n-2}{2} \alpha|x|^{2}+V \cdot x\right)=0, \forall x \in D .
$$


Consequently, for some $\bar{x} \in \mathbb{R}^{n}$ and $d \in \mathbb{R}$,

$$
v(x)^{-\frac{2}{n-2}} \equiv \alpha^{-\frac{2}{n-2}}|x-\bar{x}|^{2}+d \alpha^{-\frac{2}{n-2}} .
$$

Since $v>0$, we must have $d>0$, so

$$
v(x) \equiv\left(\frac{\alpha^{\frac{2}{n-2}}}{d+|x-\bar{x}|^{2}}\right)^{\frac{n-2}{2}} .
$$

We have proved that $v$ is of the form (11) for some $\bar{x} \in \mathbb{R}^{n}$ and some positive constants $a$ and $b$.

To finish the proof, we show that $f\left(2 b^{2} a^{-2}, \ldots, 2 b^{2} a^{-2}\right)=1$ when (18) and (19) are in effect. For $\delta>0$, let

$$
v_{\delta}(x)=v(x)-\delta|x|^{2} .
$$

Since $v_{k} \rightarrow v$ in $C^{0}\left(\bar{B}_{\delta}(0)\right)$, there exists $\beta_{k} \rightarrow 0$ and $x_{k} \rightarrow 0$ such that $\hat{v}_{k}:=v_{\delta}+\beta_{k}$ satisfies

$$
\left(v_{k}-\hat{v}_{k}\right)\left(x_{k}\right)=0 \text { and } v_{k}-\hat{v}_{k} \geq 0 \text { near } x_{k} .
$$

We have $A^{\hat{v}_{k}}\left(x_{k}\right) \geq A^{v_{k}}\left(x_{k}\right)$. Therefore, by (19), $\lambda\left(A^{\hat{v}_{k}}\left(x_{k}\right)\right) \in \Gamma$, and by (15),

$$
f\left(\lambda\left(A^{\hat{v}_{k}}\left(x_{k}\right)\right)\right) \geq f\left(\lambda\left(A^{v_{k}}\left(x_{k}\right)\right)\right)=1 .
$$

Noting that $A^{\hat{v}_{k}}\left(x_{k}\right) \rightarrow 2 b^{2} a^{-2} I$ as $\delta \rightarrow 0, k \rightarrow \infty$, we infer that $2 b^{2} a^{-2}>t_{0}$. (Indeed, if $2 b^{2} a^{-2} \leq t_{0}$, then, for small $\rho>0$, we have $A^{\hat{v}_{k}}\left(x_{k}\right)<\left(t_{0}+\rho\right) I$ for small $\delta$ and large $k$, which implies, by (44), (14) and (15), that $1 \leq f\left(\lambda\left(A^{\hat{v}_{k}}\left(x_{k}\right)\right)<f\left(t_{0}+\rho, \ldots, t_{0}+\rho\right)\right.$, which contradicts (18).) In view of (19), this implies that $\left(2 b^{2} a^{-2}, \ldots, 2 b^{2} a^{-2}\right) \in \Gamma$. We can now send $k \rightarrow \infty$ and then $\delta \rightarrow 0$ in (44) to obtain

$$
f\left(\lambda\left(A^{v}(0)\right)\right) \geq 1 \text {, i.e. } f\left(2 b^{2} a^{-2}, \ldots, 2 b^{2} a^{-2}\right) \geq 1 .
$$

Using $v^{\delta}(x)=v(x)+\delta|x|^{2}$ instead of $v_{\delta}$ and the fact that $\left(2 b^{2} a^{-2}, \ldots, 2 b^{2} a^{-2}\right) \in \Gamma$, one can easily derive

$$
f\left(2 b^{2} a^{-2}, \ldots, 2 b^{2} a^{-2}\right) \leq 1
$$

Theorem 1.1 is established.

\subsection{Proof of Theorem 1.2}

Proof of Theorem 1.2 We start with some preparation as in the proof of Theorem 1.1. We may assume that $R_{k} \geq 5$ for all $k$.

By hypotheses, $v_{k}$ is super-harmonic and positive on $\mathbb{R}^{n} \backslash\{0\}$. Therefore, $v_{*}$ is superharmonic and non-negative on $\mathbb{R}^{n} \backslash\{0\}$. Hence either $v_{*} \equiv 0$ or $v_{*}>0$ in $\mathbb{R}^{n} \backslash\{0\}$. In the former case we are done. We assume henceforth that the latter holds.

Now, for every $\beta>2$, there exists some positive constant $C(\beta)$, independent of $k$, such that $C(\beta)^{-1} \leq M_{k} v_{k} \leq C(\beta)$ in $B_{\beta}(0) \backslash B_{1 / \beta}(0)$. It follows from Theorem 2.1 that $\left|\nabla \ln v_{k}\right| \leq C^{\prime}(\beta)$ in $B_{\beta / 2}(0) \backslash B_{2 / \beta}(0)$. It follows, after passing to a subsequence, that for every $0<\alpha<1, M_{k} v_{k} \rightarrow v_{*}$ in $C_{l o c}^{\alpha}\left(\mathbb{R}^{n} \backslash\{0\}\right), v_{*} \in C_{l o c}^{0,1}\left(\mathbb{R}^{n} \backslash\{0\}\right)$ and

$$
\left|\nabla \ln v_{*}\right| \leq C^{\prime}(\beta) \text { in } B_{\beta / 2}(0) \backslash B_{2 / \beta}(0) .
$$


By the super-harmonicity and the positivity of $v_{*}$, we can find $c_{0}>0$ such that

$$
v_{*}(y) \geq 2 c_{0}|y|^{2-n}, \quad \forall|y| \geq 1 .
$$

Hence, passing to a subsequence and shrinking $R_{k}$ and $c_{0}>0$ if necessary, we can assume without loss of generality that, for all $k$,

$$
\left|M_{k} v_{k}(y)-v_{*}(y)\right| \leq\left(R_{k}\right)^{-n}, \quad \forall R_{k}^{-1} \leq|y| \leq R_{k}
$$

and

$$
M_{k} v_{k}(y) \geq c_{0}(1+|y|)^{2-n} \forall 0<|y|<R_{k}
$$

Denote

$$
\left(v_{k}\right)_{x, \lambda}(y):=\left(\frac{\lambda}{|y-x|}\right)^{n-2} v_{k}\left(x+\frac{\lambda^{2}(y-x)}{|y-x|^{2}}\right),
$$

the Kelvin transformation of $v_{k}$. We use $\left(v_{k}\right)_{\lambda}$ to denote $\left(v_{k}\right)_{0, \lambda}$.

Lemma 2.5 Under the hypotheses of Theorem 1.2, there exists a function $\lambda^{(0)}: \mathbb{R}^{n} \backslash\{0\} \rightarrow$ $(0, \infty)$ such that $\lambda^{(0)}(x) \leq|x|$ and, for all $k$,

$$
\left(v_{k}\right)_{x, \lambda} \leq v_{k} \text { in } B_{R_{k}}(0) \backslash\left(B_{\lambda}(x) \cup\{0\}\right), \forall 0<\lambda<\lambda^{(0)}(x) \text { and }|x| \leq \frac{R_{k}}{5} .
$$

Proof We adapt the proof of Lemma 2.2. For $0<|x|<\frac{R_{k}}{5}$, we have, by (47) and (48), for all $k$ that

$$
\frac{1}{c_{1}(x)} \leq M_{k} v_{k} \leq c_{1}(x) \text { in } B_{4 r_{1}(x)}(x) \subset B_{R_{k}}(0),
$$

where

$$
r_{1}(x)=\frac{1}{8}|x| \text { and } c_{1}(x)=\max \left\{1+\sup _{B_{4 r_{1}(x)}(x)} v_{*}, \frac{1}{c_{0}}(1+2|x|)^{n-2}\right\} .
$$

By Theorem 2.1 and (49), there exists $c_{2}(x)>0$, independent of $k$, such that

$$
\left|\nabla \ln v_{k}\right| \leq c_{2}(x) \text { in } B_{2 r_{1}(x)}(x) .
$$

Thus, by Lemma 2.1, we can find $0<\lambda_{1}(x)<r_{1}(x)$ independent of $k$ such that

$$
\left(v_{k}\right)_{x, \lambda} \leq v_{k} \text { in } B_{\lambda_{1}(x)}(x) \backslash\left(B_{\lambda}(x) \cup\{0\}\right) \text { for all } 0<\lambda<\lambda_{1}(x) .
$$

For $0<\lambda<\lambda_{1}(x)$, we have, using (49), that

$$
\left(v_{k}\right)_{x, \lambda}(y) \leq \frac{\lambda^{n-2} c_{1}(x)}{|y-x|^{n-2}} \text { for } y \in B_{R_{k}}(0) \backslash\left(B_{\lambda}(x) \cup\{0\}\right) .
$$

For $y \in B_{R_{k}}(0) \backslash\left(B_{1+4|x|}(x) \cup\{0\}\right)$, we have $\frac{1}{2}(1+|y|) \leq|y-x|$ and we obtain, using (51) and (48), that

$$
\left(v_{k}\right)_{x, \lambda}(y) \leq \frac{(2 \lambda)^{n-2} c_{1}(x)}{c_{0}} v_{k}(y) .
$$

For $y \in B_{1+4|x|}(x) \backslash\left(B_{\lambda_{1}(x)}(x) \cup\{0\}\right)$, we have $1+|y| \leq 2(1+3|x|),|y-x| \geq \lambda_{1}(x)$ and we obtain, using (51) and (48), that

$$
\left(v_{k}\right)_{x, \lambda}(y) \leq \frac{(2 \lambda)^{n-2} c_{1}(x)(1+3|x|)^{n-2}}{c_{0} \lambda_{1}(x)^{n-2}} v_{k}(y) .
$$


Letting

$$
\lambda^{(0)}(x)=\min \left\{\lambda_{1}(x), \frac{\lambda_{1}(x)}{2(1+3|x|)}\left[\frac{c_{1}(x)}{c_{0}}\right]^{\frac{1}{n-2}}\right\} \leq \lambda_{1}(x),
$$

we see that the conclusion of Lemma 2.5 follows from (50), (52) and (53).

Define, for $0<|x| \leq R_{k} / 5$, that

$\bar{\lambda}_{k}(x)=\sup \left\{0<\mu \leq \min \left(|x|, \frac{R_{k}}{5}\right) \mid\left(v_{k}\right)_{x, \lambda} \leq v_{k}\right.$ in $\left.B_{R_{k}}(0) \backslash\left(B_{\lambda}(x) \cup\{0\}\right), \forall 0<\lambda<\mu\right\}$.

By Lemma 2.5,

$$
\bar{\lambda}(x):=\liminf _{k \rightarrow \infty} \bar{\lambda}_{k}(x) \in\left[\lambda^{(0)}(x),|x|\right], \quad x \in \mathbb{R}^{n} \backslash\{0\} .
$$

Clearly,

$$
\left(v_{*}\right)_{x, \bar{\lambda}(x)} \leq v_{*} \text { in } \mathbb{R}^{n} \backslash\left(B_{\bar{\lambda}(x)}(x) \cup\{0\}\right) \text { for all } x \in \mathbb{R}^{n} \backslash\{0\} .
$$

We have a dichotomy:

$$
\begin{aligned}
& \text { either } \bar{\lambda}(x)=|x| \text { for all } x \in \mathbb{R}^{n} \backslash\{0\}, \\
& \quad \text { or } \bar{\lambda}\left(x_{0}\right)<\left|x_{0}\right| \text { for some } x_{0} \in \mathbb{R}^{n} \backslash\{0\} .
\end{aligned}
$$

In case (54), we obtain that $v_{*}$ is radially symmetric about the origin thanks to Lemma C.1. To finish the proof, we assume in the rest of the argument that (55) holds and derive a contradiction.

We first collect some properties of $\bar{\lambda}(x)$. We start with an analogue of Lemma 2.3. By (46), let

$$
\alpha:=\liminf _{|y| \rightarrow \infty}|y|^{n-2} v_{*}(y) \in(0, \infty]
$$

Lemma 2.6 Under the hypotheses of Theorem 1.2, if $\bar{\lambda}(x)<|x|$ for some $x \in \mathbb{R}^{n} \backslash\{0\}$, then

$$
\alpha=\lim _{|y| \rightarrow \infty}|y|^{n-2}\left(v_{*}\right)_{x, \bar{\lambda}(x)}(y)=\bar{\lambda}(x)^{n-2} v_{*}(x)<\infty .
$$

Proof We adapt Step 1 in the proof of Lemma 2.3. Assume that $\bar{\lambda}(x)<|x|$ and (without loss of generality) that $\bar{\lambda}_{k}(x) \rightarrow \bar{\lambda}(x)$. Arguing as before but using the strong maximum principle for solutions with isolated singularities [10, Theorem 1.6] instead of the standard strong maximum principle, this leads to the existence of some $y_{k} \in \partial B_{R_{k}}(0)$ such that

$$
\left(v_{k}\right)_{x, \bar{\lambda}_{k}(x)}\left(y_{k}\right)=v_{k}\left(y_{k}\right)
$$

It follows that

$$
\lim _{k \rightarrow \infty}\left|y_{k}\right|^{n-2} M_{k} v_{k}\left(y_{k}\right)=\lim _{k \rightarrow \infty}\left|y_{k}\right|^{n-2}\left(M_{k} v_{k}\right)_{x, \bar{\lambda}_{k}(x)}\left(y_{k}\right)=(\bar{\lambda}(x))^{n-2} v_{*}(x) .
$$

This implies, in view of (47), that

$$
\alpha \leq \lim _{k \rightarrow \infty}\left|y_{k}\right|^{n-2} v_{*}\left(y_{k}\right)=\bar{\lambda}(x)^{n-2} v_{*}(x)=\lim _{|y| \rightarrow \infty}|y|^{n-2}\left(v_{*}\right)_{x, \bar{\lambda}(x)}(y)<\infty .
$$

On the other hand, as in the proof of Lemma 2.3, we can use $\left(v_{*}\right)_{x, \bar{\lambda}(x)} \leq v_{*}$ in $\mathbb{R}^{n} \backslash\left(B_{\bar{\lambda}(x)}(x) \cup\right.$ $\{0\})$ to show that 


$$
\alpha \geq \bar{\lambda}(x)^{n-2} v_{*}(x)
$$

The conclusion is readily seen.

Lemma 2.7 Under the hypotheses of Theorem 1.2, if $\bar{\lambda}\left(x_{0}\right)<\left|x_{0}\right|$ for some $x_{0} \in \mathbb{R}^{n} \backslash\{0\}$, then

$$
\limsup _{x \rightarrow x_{0}} \bar{\lambda}(x) \leq \bar{\lambda}\left(x_{0}\right)
$$

Proof Along a subsequence, we have $\bar{\lambda}_{k}\left(x_{0}\right) \rightarrow \bar{\lambda}\left(x_{0}\right)$.

As in the proof of Lemma 2.6, there exists $y_{k} \in \partial B_{R_{k}}(0)$ such that

$$
\left(v_{k}\right)_{x_{0}, \bar{\lambda}_{k}\left(x_{0}\right)}\left(y_{k}\right)=v_{k}\left(y_{k}\right)
$$

We know that

$$
\eta_{k}:=\sup _{B\left|x_{0}\right| / 2\left(x_{0}\right)}\left|M_{k} v_{k}-v_{*}\right| \rightarrow 0 \text { as } k \rightarrow \infty .
$$

Let $m$ denote the modulus of continuity of $v_{*}$ in $B_{\left|x_{0}\right| / 2}\left(x_{0}\right)$, i.e.

$$
m(r)=\sup \left\{\left|v_{*}(x)-v_{*}(y)\right|: x, y \in B_{\left|x_{0}\right| / 2}\left(x_{0}\right),|x-y|<r\right\} .
$$

In the computation below, we use $o(1)$ to denote quantities such that

$$
\lim _{k \rightarrow \infty} o(1)=0 .
$$

Fix some $\delta>0$ and consider $\left|x-x_{0}\right|<\left|x_{0}\right| / 2$. We note that

$$
\left|\left(x+\frac{\left(\bar{\lambda}_{k}\left(x_{0}\right)+\delta\right)^{2}\left(y_{k}-x\right)}{\left|y_{k}-x\right|^{2}}\right)-\left(x_{0}+\frac{\bar{\lambda}_{k}\left(x_{0}\right)^{2}\left(y_{k}-x_{0}\right)}{\left|y_{k}-x_{0}\right|^{2}}\right)\right|=\left|x-x_{0}\right|+o(1) .
$$

Thus,

$$
\left|v_{*}\left(x+\frac{\left(\bar{\lambda}_{k}\left(x_{0}\right)+\delta\right)^{2}\left(y_{k}-x\right)}{\left|y_{k}-x\right|^{2}}\right)-v_{*}\left(x_{0}+\frac{\bar{\lambda}_{k}\left(x_{0}\right)^{2}\left(y_{k}-x_{0}\right)}{\left|y_{k}-x_{0}\right|^{2}}\right)\right| \leq m\left(\left|x-x_{0}\right|+o(1)\right) .
$$

It follows that

$$
\begin{aligned}
& M_{k}\left(v_{k}\right)_{x, \bar{\lambda}_{k}\left(x_{0}\right)+\delta}\left(y_{k}\right)=\left(\frac{\bar{\lambda}_{k}\left(x_{0}\right)+\delta}{\left|y_{k}-x\right|}\right)^{n-2}\left(M_{k} v_{k}\right)\left(x+\frac{\left(\bar{\lambda}_{k}\left(x_{0}\right)+\delta\right)^{2}\left(y_{k}-x\right)}{\left|y_{k}-x\right|^{2}}\right) \\
& \geq\left(\frac{\bar{\lambda}_{k}\left(x_{0}\right)+\delta}{\left|y_{k}-x\right|}\right)^{n-2}\left[\left(M_{k} v_{k}\right)\left(x_{0}+\frac{\bar{\lambda}_{k}\left(x_{0}\right)^{2}\left(y_{k}-x_{0}\right)}{\left|y_{k}-x_{0}\right|^{2}}\right)\right. \\
& \left.-2 \eta_{k}-m\left(\left|x-x_{0}\right|+o(1)\right)\right] \\
& =\left(1+\frac{\delta}{\bar{\lambda}_{k}\left(x_{0}\right)}\right)^{n-2} M_{k}\left(v_{k}\right)_{x_{0}, \bar{\lambda}_{k}\left(x_{0}\right)}\left(y_{k}\right) \\
& -\left(\frac{\bar{\lambda}_{k}\left(x_{0}\right)+\delta}{\left|y_{k}-x\right|}\right)^{n-2}\left[2 \eta_{k}+m\left(\left|x-x_{0}\right|+o(1)\right)\right] .
\end{aligned}
$$

Recalling (56), we arrive at

$$
\begin{aligned}
M_{k}\left(v_{k}\right)_{x, \bar{\lambda}_{k}\left(x_{0}\right)+\delta}\left(y_{k}\right) \geq(1+ & \left.\frac{\delta}{\bar{\lambda}_{k}\left(x_{0}\right)}\right)^{n-2} M_{k} v_{k}\left(y_{k}\right) \\
& -\left(\frac{\bar{\lambda}_{k}\left(x_{0}\right)+\delta}{\left|y_{k}\right|}\right)^{n-2}\left[o(1)+m\left(\left|x-x_{0}\right|+o(1)\right)\right] .
\end{aligned}
$$


Thus, in view of (48), we can find small $\bar{\epsilon}>0$ depending only on $\delta, c, \bar{\lambda}\left(x_{0}\right)$ and the function $m(\cdot)$ such that, for all $\left|x-x_{0}\right|<\bar{\epsilon}$ and for large $k$,

$$
M_{k}\left(v_{k}\right)_{x, \bar{\lambda}_{k}\left(x_{0}\right)+\delta}\left(y_{k}\right) \geq\left(1+\frac{\delta}{4 \bar{\lambda}_{k}\left(x_{0}\right)}\right)^{n-2} M_{k} v_{k}\left(y_{k}\right) .
$$

This implies that (cf. (56)), that

$$
\bar{\lambda}_{k}(x) \leq \bar{\lambda}_{k}\left(x_{0}\right)+\delta \text { for all }\left|x-x_{0}\right|<\bar{\epsilon} \text { and large } k .
$$

The conclusion follows.

We now return to drawing a contradiction from (55). By Lemma 2.7, we infer from (55) that there exists some $r_{0}>0$ such that $\bar{\lambda}(x)<|x|$ for all $x \in B_{r_{0}}\left(x_{0}\right)$. We can then argue as in the proof of Theorem 1.1, using Lemma 2.6 instead of Lemma 2.3 to obtain

$$
v_{*}(x)=\left(\frac{a}{1+b^{2}|x-\bar{x}|^{2}}\right)^{\frac{n-2}{2}} \quad x \in B_{r_{0}}\left(x_{0}\right) .
$$

for some $\bar{x} \in \mathbb{R}^{n}$ and some $a, b>0$. For small $\delta>0$, let

$$
v_{*}^{\delta}(x):=v_{*}(x)+\delta\left|x-x_{0}\right|^{2} .
$$

Since $M_{k} v_{*} \rightarrow v_{*}$ in $C^{0}\left(\bar{B}_{\delta}\left(x_{0}\right)\right)$, there exists $\beta_{k} \rightarrow 0$ and $x_{k} \rightarrow x_{0}$ such that the function $\xi_{k, \delta}:=v_{*}^{\delta}+\beta_{k}$ satisfies

$$
\left(M_{k} v_{k}-\xi_{k, \delta}\right)\left(x_{k}\right)=0 \text { and } M_{k} v_{k}-\xi_{k, \delta} \leq 0 \text { near } x_{k} .
$$

It follows that

$$
A^{v_{k}}\left(x_{k}\right) \geq A^{\frac{1}{M_{k}} \xi_{k, \delta}}\left(x_{k}\right)=M_{k}^{\frac{4}{n-2}} A^{\xi_{k, \delta}}\left(x_{k}\right) .
$$

On the other hand, by hypothesis, there is some $\lambda_{*} \in \Gamma$ such that $f\left(\lambda_{*}\right)=1$ (e.g. $\lambda_{*}=$ $\left.\lambda\left(A^{v_{1}}(0)\right)\right)$. By (15), we can find $\hat{\lambda}_{*} \in \Gamma$ such that $f\left(\hat{\lambda}_{*}\right)>1$. As $M_{k} \rightarrow \infty$ and $A^{\xi, \delta}\left(x_{k}\right)=$ $2 b^{2} a^{-2} I+O(\delta)$, we can find $k$ sufficiently large such that $M_{k}^{\frac{4}{n-2}} A^{\xi k, \delta}\left(x_{k}\right)>\operatorname{diag}\left(\hat{\lambda}_{*}\right)$. We are thus led to

$$
A^{v_{k}}\left(x_{k}\right)>\operatorname{diag}\left(\hat{\lambda}_{*}\right) .
$$

As $f\left(\lambda\left(A^{v_{k}}\right)\right)=1$ and $f\left(\hat{\lambda}_{*}\right)>1$, the above contradicts (14) and (15).

\section{Local gradient estimates}

In this section, we adapt the argument in [12] to prove Theorem 1.6.

For a locally Lipschitz function $w$ in $B_{2}(0), 0<\alpha<1, x \in B_{2}(0)$ and $0<\delta<2-|x|$, define

$$
\begin{aligned}
{[w]_{\alpha, \delta}(x) } & =\sup _{0<|y-x|<\delta} \frac{|w(y)-w(x)|}{|y-x|^{\alpha}} \\
\delta(w, x, \alpha) & = \begin{cases}\infty & \text { if }(2-|x|)^{\alpha}[w]_{\alpha, 2-|x|}(x)<1, \\
\mu & \text { where } 0<\mu \leq 2-|x| \text { and } \mu^{\alpha}[w]_{\alpha, \mu}(x)=1 \\
& \text { if }(2-|x|)^{\alpha}[w]_{\alpha, 2-|x|}(x) \geq 1 .\end{cases}
\end{aligned}
$$

Note that $\delta(w, x, \alpha)$ is well defined as $[w]_{\alpha, \delta}(x)$ is continuous and non-decreasing in $\delta$. The object $\delta(w, x, \alpha)$ was introduced in [12]. Its reciprocal $\delta(w, x, \alpha)^{-1}$ plays a role similar to 
that of $|\nabla w(x)|$ in performing a rescaling argument for a sequence of functions blowing up in $C^{\alpha}$-norms. For example, when $\delta=\delta(w, x, \alpha)<\infty$, the rescaled function $\hat{w}(y):=$ $w(x+\delta y)-w(x)$ satisfies

$$
\hat{w}(0)=0 \text { and }[\hat{w}]_{\alpha, 1}(0)=\delta^{\alpha}[\hat{w}]_{\alpha, \delta}(x)=1 .
$$

Proof of Theorem 1.6 By the conformal invariance (7), it suffices to show bound $|\nabla \ln v|$ in $B_{1 / 4}(0)$.

We first claim that

$$
\sup _{x \neq y \in B_{1 / 2}(0)} \frac{|\ln v(x)-\ln v(y)|}{|x-y|^{\alpha}} \leq C(\Gamma, \alpha) \text { for any } 0<\alpha<1 .
$$

Assume otherwise that (57) fails for some $0<\alpha<1$. Then there exist $0<v_{i} \in$ $C^{2}\left(B_{2}(0)\right)$ such that $f\left(\lambda\left(A^{v_{i}}\right)\right)=1$ and $v_{i} \leq b$ in $B_{2}(0)$ but

$$
\sup _{x \neq y \in B_{1 / 2}(0)} \frac{\left|\ln v_{i}(x)-\ln v_{i}(y)\right|}{|x-y|^{\alpha}} \rightarrow \infty .
$$

This implies that, for any fixed $0<r<1 / 2$,

$$
\sup _{x \in B_{1 / 2}(0)}\left[\ln v_{i}\right]_{\alpha, r}(x) \rightarrow \infty \text { and } \inf _{x \in B_{1 / 2}(0)} \delta\left(\ln v_{i}, x, \alpha\right) \rightarrow 0 .
$$

Therefore, there exists $x_{i} \in B_{1}(0)$,

$$
\frac{1-\left|x_{i}\right|}{\delta\left(\ln v_{i}, x_{i}, \alpha\right)}=\sup _{x \in B_{1}(0)} \frac{1-|x|}{\delta\left(\ln v_{i}, x, \alpha\right)} \rightarrow \infty .
$$

Let $\sigma_{i}=\frac{1-\left|x_{i}\right|}{2}$ and $\epsilon_{i}=\delta\left(\ln v_{i}, x_{i}, \alpha\right)$. Then

$$
\frac{\sigma_{i}}{\epsilon_{i}} \rightarrow \infty, \epsilon_{i} \rightarrow 0, \text { and } \epsilon_{i} \leq 2 \delta\left(\ln v_{i}, z, \alpha\right) \text { for any }\left|z-x_{i}\right| \leq \sigma_{i} .
$$

We now define

$$
\hat{v}_{i}(y)=\frac{1}{v_{i}\left(x_{i}\right)} v_{i}\left(x_{i}+\epsilon_{i} y\right) \text { for }|y| \leq \frac{\sigma_{i}}{\epsilon_{i}} .
$$

Then

$$
\left[\ln \hat{v}_{i}\right]_{\alpha, 1}(0)=\epsilon_{i}^{\alpha}\left[\ln v_{i}\right]_{\alpha, \epsilon_{i}}\left(x_{i}\right)=1 .
$$

Also, by (58), for any fixed $\beta>1$ and $|y|<\beta$, there holds

$$
\begin{aligned}
{\left[\ln \hat{v}_{i}\right]_{\alpha, 1}(y) } & =\epsilon_{i}^{\alpha}\left[\ln v_{i}\right]_{\alpha, \epsilon_{i}}\left(x_{i}+\epsilon_{i} y\right) \\
& \leq 2^{-\alpha} \epsilon_{i}^{\alpha}\left\{\sup _{\left|z-\left(x_{i}+\epsilon_{i} y\right)\right| \leq \epsilon_{i}}\left[\ln v_{i}\right]_{\alpha, \epsilon_{i} / 4}(z)+\left[\ln v_{i}\right]_{\alpha, \epsilon_{i} / 4}\left(x_{i}+\epsilon_{i} y\right)\right\} \\
& \leq \sup _{\left|z-\left(x_{i}+\epsilon_{i} y\right)\right| \leq \epsilon_{i}} \delta\left(\ln v_{i}, z, \alpha\right)^{\alpha}\left[\ln v_{i}\right]_{\alpha, \delta\left(\ln v_{i}, z, \alpha\right)}(z) \\
& \quad+\delta\left(\ln v_{i}, x_{i}+\epsilon_{i} y, \alpha\right)^{\alpha}\left[\ln v_{i}\right]_{\alpha, \delta\left(\ln v_{i}, x_{i}+\epsilon_{i} y, \alpha\right)}\left(x_{i}+\epsilon_{i} y\right) \\
& =2
\end{aligned}
$$

for all sufficiently large $i$. Since $\hat{v}_{i}(0)=1$ by definition, we deduce from (59) and (60) that

$$
\frac{1}{C(\beta)} \leq \hat{v}_{i}(y) \leq C(\beta) \text { for }|y| \leq \beta \text { and all sufficiently large } i .
$$


We can now apply Theorem 2.1 to obtain

$$
\left|\nabla \ln \hat{v}_{i}\right| \leq C(\beta) \text { in } B_{\beta / 2}(0) \text { for all sufficiently large } i .
$$

Passing to a subsequence and recalling (58) and (61), we see that $\hat{v}_{i}$ converges in $C^{0, \alpha^{\prime}}$ $\left(\alpha<\alpha^{\prime}<1\right)$ on compact subsets of $\mathbb{R}^{n}$ to some positive, locally Lipschitz function $v_{*}$.

On the other hand, if we define

$$
\bar{v}_{i}(y)=\epsilon_{i}^{\frac{n-2}{2}} v_{i}\left(x_{i}+\epsilon_{i} y\right) \text { for }|y| \leq \frac{\sigma_{i}}{\epsilon_{i}},
$$

then by the conformal invariance (7), we have

$$
f\left(\lambda\left(A^{\bar{v}_{i}}\right)\right)=1 \text { in } B_{\sigma_{i} / \epsilon_{i}}(0) .
$$

Since $\frac{\sigma_{i}}{\epsilon_{i}} \rightarrow \infty, \hat{v}_{i}=M_{i} \bar{v}_{i}$ where $M_{i}=v_{i}\left(x_{i}\right)^{-1} \epsilon_{i}^{-\frac{n-2}{2}} \rightarrow \infty\left(\right.$ thanks to the bound $\left.v_{i} \leq b\right)$, we then conclude from Theorem 1.2 that $v_{*}$ is constant, namely

$$
v_{*} \equiv v_{*}(0)=\lim _{i \rightarrow \infty} \hat{v}_{i}(0)=1 .
$$

This contradicts (59), in view of (62) and the convergence of $\hat{v}_{i}$ to $v_{*}$. We have proved (57).

From (57), we can find some universal constant $C>1$ such that

$$
\frac{u(0)}{C} \leq u \leq C u(0) \text { in } B_{1 / 2}(0) \text {. }
$$

Applying Theorem 2.1 again we obtain the required gradient estimate in $B_{1 / 4}(0)$.

\section{Fine blow-up analysis}

\subsection{A quantitative centered Liouville-type result}

In this subsection, we establish:

Proposition 4.1 Let $(f, \Gamma)$ satisfy (13)-(15), (18)-(19), (5) and the normalization condition (21). Assume that for a sequence $R_{k} \rightarrow \infty, 0<v_{k} \in C^{2}\left(B_{R_{k}}\right)$ satisfy

$$
f\left(\lambda\left(A^{v_{k}}\right)\right)(y)=1, \quad 0<v_{k}(y) \leq v_{k}(0)=1, \quad|y| \leq R_{k} .
$$

Then for every $\epsilon>0$, there exists a constant $\delta_{0}>0$, depending only on $(f, \Gamma)$ and $\epsilon$, such that, for all sufficiently large $k$,

$$
\left|v_{k}(y)-U(y)\right| \leq 2 \epsilon U(y), \quad \forall|y| \leq \delta_{0} R_{k} .
$$

Recall that $U=\left(1+|x|^{2}\right)^{-\frac{n-2}{2}}, A^{U} \equiv 2 I$ and $f\left(\lambda\left(A^{U}\right)\right)=1$ on $\mathbb{R}^{n}$.

Proposition 4.1 is equivalent to the following proposition.

Proposition 4.2 Let ( $f, \Gamma$ ) satisfy (13)-(15), (18)-(19), (5) and the normalization condition (21). For any $\epsilon>0$ there exist $\delta_{0}, C_{0}>0$ depending only on $(f, \Gamma)$ and $\epsilon$ such that if $0<u \in C^{2}\left(B_{R}(0)\right), R>0$, satisfies

$$
f\left(\lambda\left(A^{u}\right)\right)=1 \text { in } B_{R}(0) \text { and } u(0)=\sup _{B_{R}(0)} u \geq C_{0} R^{-\frac{n-2}{2}},
$$

then

$$
\left|u(x)-U^{0, u(0)}(x)\right| \leq 2 \epsilon U^{0, u(0)}(x) \text { for all } x \in B_{\delta_{0} R}(0) .
$$


Proof of the equivalence between Propositions 4.1 and 4.2 It is clear that Proposition 4.2 implies Proposition 4.1.

Consider the converse. Let $\delta_{0}=\delta_{0}(\epsilon)$ be as in Proposition 4.1. Arguing by contradiction, we assume that there are some $\epsilon>0$ and a sequence of $R_{k}$ and $u_{k} \in C^{2}\left(B_{R_{k}}(0)\right)$ such that

$$
f\left(\lambda\left(A^{u_{k}}\right)\right)=1 \text { in } B_{R_{k}}(0) \text { and } u_{k}(0)=\sup _{B_{R_{k}}(0)} u_{k} \geq k R_{k}^{-\frac{n-2}{2}}
$$

but the last estimate in Proposition 4.2 fails for each $k$.

Define

$$
\bar{u}_{k}(y)=\frac{1}{u_{k}(0)} u_{k}\left(\frac{y}{u_{k}(0)^{\frac{2}{n-2}}}\right) \text { for }|y| \leq R_{k} u_{k}(0)^{\frac{2}{n-2}}=: \bar{R}_{k} .
$$

Then $f\left(\lambda\left(A^{\bar{u}_{k}}\right)\right)=1$ in $B_{\bar{R}_{k}}(0), \sup _{B_{\bar{R}_{k}}(0)} \bar{u}_{k}=\bar{u}_{k}(0)=1$, and $\bar{R}_{k} \geq k^{\frac{2}{n-2}} \rightarrow \infty$. By Proposition 4.1,

$$
\left|\bar{u}_{k}(y)-U(y)\right| \leq 2 \epsilon U(y) \text { in } B_{\delta_{0}} \bar{R}_{k}(0) \text { for all sufficiently large } k .
$$

Returning to the original sequence $u_{k}$ we arrive at a contradiction.

Lemma 4.1 Under the hypotheses of Proposition 4.1 except for (5), we have

$$
v_{k} \rightarrow U, \quad \text { in } C_{l o c}^{\beta}\left(\mathbb{R}^{n}\right), \quad \forall 0<\beta<1 .
$$

Moreover, for every $\epsilon>0$, there exists $k_{0} \geq 1$ such that

$$
\min _{|y|=r} v_{k}(y) \leq(1+\epsilon) U(r), \quad \forall 0<r<R_{k} / 5, k \geq k_{0} .
$$

Proof We first prove (65). Since $v_{k}$ satisfies (63), we deduce from Theorem 1.6 that

$$
\left|\nabla \ln v_{k}\right| \leq C \quad \text { in } B_{R_{k}-1}, \quad \forall k,
$$

where $C$ is independent of $k$. This yields (65) in view of Theorem 1.1.

We now prove (66). Suppose the contrary, then there exists some $\epsilon>0$ and sequences of $k_{i} \rightarrow \infty, 0<r_{i}<R_{k_{i}} / 5$ such that

$$
v_{k_{i}}>(1+\epsilon) U \text { on } \partial B_{r_{i}} .
$$

Because of (65), $r_{i} \rightarrow \infty$.

As in the proof of Lemma 2.2, there exists $\lambda_{i}^{(0)}>0$ such that

$$
\left(v_{k_{i}}\right)_{\lambda} \leq v_{k_{i}} \text { in } B_{r_{i}} \backslash B_{\lambda}, \forall 0<\lambda<\lambda_{i}^{(0)} \text { and }|x| \leq r_{i} .
$$

By the explicit expression of $U$, there exists some small $\delta>0$ independent of $i$ such that, for large $i$,

$$
U_{\lambda}(y) \leq\left(1+\frac{\epsilon}{4}\right) U(y), \quad \forall y \in \partial B_{r_{i}}, \lambda_{i}^{(0)} \leq \lambda \leq 1+\delta,
$$

By the uniform convergence of $v_{k_{i}}$ to $U$ on compact subsets of $\mathbb{R}^{n}$, we have, for large $i$,

$$
\left(v_{k_{i}}\right) \lambda \leq\left(1+\frac{\epsilon}{2}\right) U(y), \quad \forall y \in \partial B_{r_{i}}, \lambda_{i}^{(0)} \leq \lambda \leq 1+\delta,
$$


As in the proof of Lemma 2.2, the moving sphere procedure does not stop before reaching $\lambda=1+\delta$, namely we have, for large $i$,

$$
\left(v_{k_{i}}\right)_{\lambda} \leq v_{k_{i}} \text { in } B_{r_{i}} \backslash B_{\lambda}, \forall 0<\lambda<1+\delta \text { and }|x| \leq r_{i} .
$$

Sending $i$ to $\infty$ leads to

$$
U_{1+\delta}(y) \leq U(y), \quad \forall 1+\delta \leq|y| \leq 2 .
$$

A contradiction-since we see from the explicit expression of $U$ that $U_{1+\delta}(y)>U(y)$ for all $1<1+\delta<|y| \leq 2$.

Lemma 4.2 Under the hypotheses of Proposition 4.1, for any $\epsilon>0$, there exist a small $\delta_{1}>0$ and a large $r_{1}>1$, depending only on $(f, \Gamma)$ and $\epsilon$, such that, for all sufficiently large $k$,

$$
\begin{array}{ll} 
& v_{k}(y) \geq(1-\epsilon) U(y), \quad \forall|y| \leq \delta_{1} R_{k}, \\
\text { and } \quad \int_{r_{1} \leq|y| \leq \delta_{1} R_{k}} v_{k}^{\frac{n+2}{n-2}} \leq \epsilon .
\end{array}
$$

Proof Assume without loss of generality that $\epsilon \in(0,1 / 2)$. Since $v_{k} \rightarrow U$ in $C_{l o c}^{0}\left(\mathbb{R}^{n}\right)$, there exist $r_{2}>1$ and $k_{1}$, depending on $\epsilon$, such that for all $k \geq k_{1}$

$$
\begin{aligned}
& v_{k}(y) \geq\left(1-\epsilon^{2}\right) U(y), \quad \forall|y| \leq r_{2}, \\
& v_{k}(y) \geq\left(1-\epsilon^{2}\right) U\left(r_{2}\right) \geq\left(1-2 \epsilon^{2}\right) r_{2}^{2-n}, \quad \forall|y|=r_{2} .
\end{aligned}
$$

By (5),

$$
\operatorname{Trace}\left(A^{v_{k}}\right) \geq \delta>0 \text {, }
$$

and therefore

$$
-\Delta v_{k}(y) \geq \frac{n-2}{2} \delta v_{k}(y)^{\frac{n+2}{n-2}} \quad \text { in } r_{2} \leq|y| \leq R_{k} .
$$

Using the superharmonicity of $v_{k}$ and the maximum principle, we obtain

$$
v_{k}(y) \geq\left(1-\epsilon^{2}\right)\left(|y|^{2-n}-R_{k}^{2-n}\right), \quad r_{2} \leq|y| \leq R_{k} .
$$

Thus, for any $\delta_{2} \in\left(0, \epsilon^{\frac{2}{n-2}}\right)$, we have for all sufficiently large $k$ that

$$
v_{k}(y) \geq\left(1-\epsilon^{2}\right)\left(1-\delta_{2}^{n-2}\right)|y|^{2-n} \geq\left(1-2 \epsilon^{2}\right)|y|^{2-n}, \quad r_{2} \leq|y| \leq \delta_{2} R_{k} .
$$

Now if $\delta_{1}<\delta_{2}$, (69) is readily seen from (71) and (74).

Let

$$
\hat{v}_{k}(y):=v_{k}(y)-\left(1-2 \epsilon^{2}\right)|y|^{2-n} .
$$

Then

$$
-\Delta \hat{v}_{k}(y) \geq \hat{f}(y):=\frac{n-2}{2} \delta v_{k}(y)^{\frac{n+2}{n-2}} \quad \text { in } r_{2} \leq|y| \leq \delta_{2} R_{k},
$$

and

$$
\hat{v}_{k}(y) \geq 0, \quad \text { for } y \in \partial\left(B_{\delta_{2} R_{k}} \backslash B_{r_{2}}\right)
$$


Let $R_{k}^{\prime}=\frac{\delta_{2} R_{k}}{2}$. Enlarging $k_{1}$ if necessary, we can apply Corollary A.3 in "Appendix A" to get

$$
\min _{|x|=R_{k}^{\prime}} \hat{v}_{k}(x) \geq C^{-1}\left(\delta_{2} R_{k}\right)^{2-n} \int_{2 r_{2} \leq|y| \leq \delta_{2} R_{k} / 8} \delta v_{k}(y)^{\frac{n+2}{n-2}} d y, \quad \forall k \geq k_{1},
$$

where here and below $C$ is some positive constant depending only on $n$. On the other hand, by Lemma 4.1, we have (after enlarging $k_{1}$ if necessary)

$$
\min _{|x|=R_{k}^{\prime}} v_{k}(x) \leq\left(1+\epsilon^{2}\right) U\left(R_{k}^{\prime}\right) \leq\left(1+2 \epsilon^{2}\right)\left(R_{k}^{\prime}\right)^{2-n}, \quad \forall k \geq k_{1},
$$

which implies that

$$
\min _{|x|=R_{k}^{\prime}} \hat{v}_{k}(x) \leq C \epsilon^{2}\left(\delta_{2} R_{k}\right)^{2-n}, \quad \forall k \geq k_{1} .
$$

It now follows from (75) and (76) that

$$
\int_{2 r_{2} \leq|y| \leq \delta_{2} R_{k} / 8} \delta v_{k}(y)^{\frac{n+2}{n-2}} d y \leq c_{1} \epsilon^{2}
$$

where $c_{1}$ depends only on $n$. (70) is then established for $\epsilon \leq \frac{1}{c_{1}}$ with $r_{1}=2 r_{2}$ and $\delta_{1}=\delta_{2} / 8$. The conclusion for $\epsilon>1 / c_{1}$ also follows.

Lemma 4.3 Let $(f, \Gamma)$ satisfy (13)-(15). Then there exist $\delta_{3}>0$ and $C_{3}>1$, depending only on $(f, \Gamma)$, such that if $u \in C^{2}\left(B_{2}(0)\right)$ satisfies

$$
f\left(\lambda\left(A^{u}\right)\right)=1, u>0, \quad \text { in } B_{2}(0),
$$

and

$$
\int_{B_{2}(0)} u^{\frac{2 n}{n-2}} \leq \delta_{3}
$$

then

$$
u \leq C_{3} \text { in } B_{1}(0)
$$

If $(f, \Gamma)$ satisfies in addition the conditions (18), (19) and the normalization condition (21), then $\delta_{3}$ can be chosen to be any constant smaller than $\int_{\mathbb{R}^{n}} U^{\frac{2 n}{n-2}} d x$.

Proof We adapt the proof of [7, Lemma 6.4]. Arguing by contradiction, we can find a sequence of $0<u_{j} \in C^{2}\left(B_{2}\right)$ such that $f\left(\lambda\left(A^{u_{j}}\right)\right)=1$ in $B_{2}(0)$,

$$
\int_{B_{2}(0)} u_{j}^{\frac{2 n}{n-2}} \rightarrow 0
$$

but

$$
d\left(y_{j}\right)^{\frac{n-2}{2}} u_{j}\left(y_{j}\right)=\max _{\bar{B}_{3 / 2}(0)} d(y)^{\frac{n-2}{2}} u_{j}(y) \rightarrow \infty,
$$

where $y_{j} \in B_{3 / 2}(0)$ and $d(y)=3 / 2-|y|$.

Let $\sigma_{j}=\frac{1}{2} d\left(y_{j}\right)>0$,

$$
v_{j}(z)=\frac{1}{u_{j}\left(y_{j}\right)} u_{j}\left(y_{j}+\frac{1}{u_{j}\left(y_{j}\right)^{\frac{2}{n-2}}} z\right) \text { for }|z|<r_{j}:=u_{j}\left(y_{j}\right)^{\frac{2}{n-2}} \sigma_{j} \rightarrow \infty .
$$


Then by the conformal invariance property (7), $f\left(\lambda\left(A^{v_{j}}\right)\right)=1$ in $B_{r_{j}}(0), v_{j}(0)=1$, $v_{j} \leq 2^{\frac{n-2}{2}}$ in $B_{r_{j}}(0)$ and

$$
\int_{B_{r_{j}}(0)} v_{j}^{\frac{2 n}{n-2}} \rightarrow 0
$$

By Theorem 1.6, there is a constant $C$ independent of $j$ such that

$$
\left|\nabla \ln v_{j}\right| \leq C \text { in } B_{r_{j} / 2}(0) .
$$

Thus, after passing to a subsequence, we can assume that $v_{j}$ converges in $C_{l o c}^{0}\left(\mathbb{R}^{n}\right)$ to some positive function $v$ (as $\left.v_{j}(0)=1\right)$. This contradicts (77).

The above argument can be adapted to prove the last assertion of the lemma: Eq. (77) is replaced by

$$
\int_{B_{r_{j}}(0)} v_{j}^{\frac{2 n}{n-2}} \leq \delta_{3}<\int_{\mathbb{R}^{n}} U^{\frac{2 n}{n-2}} d x .
$$

On the other hand, by Theorem 1.1, we have $v_{j} \rightarrow U$ in $C_{l o c}^{0}\left(\mathbb{R}^{n}\right)$. This gives a contradiction.

Lemma 4.4 Let $(f, \Gamma)$ satisfy (13)-(15) and let $\delta_{3}, C_{3}$ be as in Lemma 4.3. If $u \in C^{2}\left(B_{2 R}(0)\right)$ satisfies

$$
f\left(\lambda\left(A^{u}\right)\right)=1, u>0, \quad \text { in } B_{2 R}(0),
$$

and

$$
\int_{B_{2 R(0)}} u^{\frac{2 n}{n-2}} \leq \delta_{3}
$$

then

$$
u \leq C_{3} R^{-\frac{n-2}{2}} \text { in } B_{R}(0) .
$$

Proof This follows from Lemma 4.3 and a change of variables, $\tilde{u}(y)=R^{\frac{n-2}{2}} u(R y)$ for $|y| \leq 2$.

Lemma 4.5 Under the hypotheses of Proposition 4.1, there exist positive constants $\delta_{4}>0$ and $C_{4}>1$, depending only on $(f, \Gamma)$, such that, for all sufficiently large $k$,

$$
v_{k}(y) \leq C_{4} U(y), \quad \forall|y| \leq \delta_{4} R_{k} .
$$

Proof Let $\delta_{3}$ be as in Lemma 4.3. Since $v_{k} \leq 1$, we deduce from Lemma 4.2, there is $r_{1}>1$ and $\delta_{1}>0$ such that

$$
\int_{r_{1} \leq|y| \leq \delta_{1} R_{k}} v_{k}^{\frac{2 n}{n-2}} \leq \epsilon .
$$

For any $2 r_{1}<r<\delta_{1} R_{k} / 2$, consider

$$
\tilde{v}_{k}(z)=r^{\frac{n-2}{2}} v_{k}(r z), \quad \frac{1}{2}<|z|<2 .
$$

By (79), we have, for large $k$,

$$
\int_{\frac{1}{2}<|z|<2} \tilde{v}_{k}(z)^{\frac{2 n}{n-2}}=\int_{\frac{r}{2}<|\eta|<2 r} v_{k}(\eta)^{\frac{2 n}{n-2}} \leq \delta_{3} .
$$


It follows from Lemma 4.4 that

$$
\tilde{v}_{k}(z) \leq C \quad \forall \frac{2}{3}<|z|<\frac{5}{4},
$$

for some universal constant $C$. Since $\tilde{v}_{k}$ also satisfies $f\left(\lambda\left(A^{\tilde{v}_{k}}\right)\right)=1$, we can apply Theorem 1.6 to obtain

$$
\left|\nabla \ln \tilde{v}_{k}(z)\right| \leq C \quad \forall|z|=1,
$$

which implies that $\max _{|z|=1} \tilde{v}_{k} \leq C \min _{\partial B_{1}} \tilde{v}_{k}$. Returning to $v_{k}$, we obtain

$$
\max _{\partial B_{r}} v_{k} \leq C \min _{\partial B_{r}} v_{k}
$$

where $C$ is universal. The conclusion then follows from Lemma 4.1.

Proof of Proposition 4.1 Fix $\epsilon>0$ 0. In view of Lemma 4.2 (cf. (69)), we only need to prove that there exist $\delta_{0}>0$ such that, for all sufficiently large $k$,

$$
v_{k}(y) \leq(1+2 \epsilon) U(y), \quad \forall|y| \leq \delta_{0} R_{k} .
$$

Suppose the contrary of the above, then, after passing to a subsequence and renaming the subsequence still as $\left\{v_{k}\right\}$ and $\left\{R_{k}\right\}$, there exist $\left|y_{k}\right|=\delta_{k} R_{k}, \delta_{k} \rightarrow 0^{+}$, such that

$$
v_{k}\left(y_{k}\right)=\max _{|y|=\delta_{k} R_{k}} v_{k}(y)>(1+2 \epsilon) U\left(y_{k}\right) .
$$

In view of the convergence of $v_{k}$ to $U,\left|y_{k}\right| \rightarrow \infty$ as $k \rightarrow \infty$.

Consider the following two rescalings of $v_{k}$ :

$$
\hat{v}_{k}(z):=\left|y_{k}\right|^{n-2} v_{k}\left(\left|y_{k}\right| z\right) \text { and } \bar{v}_{k}(z)=\left|y_{k}\right|^{\frac{n-2}{2}} v_{k}\left(\left|y_{k}\right| z\right), \quad|z|<\frac{R_{k}}{\left|y_{k}\right|} \rightarrow \infty \text {. }
$$

By Lemma 4.5, we have

$$
\hat{v}_{k}(z) \leq C|z|^{2-n} \text { and } \bar{v}_{k}(z) \leq C\left|y_{k}\right|^{-\frac{n-2}{2}}|z|^{2-n}
$$

for some constant $C$ independent of $k$.

In view of the conformal invariance (7) and (63),

$$
f\left(\lambda\left(A^{\bar{v}_{k}}(z)\right)\right)=1 \text { for }|z|<\frac{R_{k}}{\left|y_{k}\right|} .
$$

Recalling (83), we can apply Theorem 1.6 to obtain that for all $0<\alpha<\beta<\infty$, there exists positive constant $C(\alpha, \beta)$ such that for large $k$,

$$
\left|\nabla \ln \bar{v}_{k}(z)\right| \leq C(\alpha, \beta), \quad \forall \alpha<|z|<\beta,
$$

which implies that

$$
\left|\nabla \ln \hat{v}_{k}(z)\right| \leq C(\alpha, \beta), \quad \forall \alpha<|z|<\beta .
$$

We know from (82), (81) and Lemma 4.1 that

$$
\min _{|z|=1} \hat{v}_{k}(z) \leq(1+\epsilon) \frac{\left|y_{k}\right|^{n-2}}{U\left(y_{k}\right)}
$$

and

$$
\max _{|z|=1} \hat{v}_{k}(z) \geq(1+2 \epsilon) \frac{\left|y_{k}\right|^{n-2}}{U\left(y_{k}\right)} .
$$


We deduce from (85), (86) and (87), after passing to a subsequence, that for some positive function $\hat{v}^{*}$ in $C_{l o c}^{0,1}\left(\mathbb{R}^{n} \backslash\{0\}\right)$,

$$
\hat{v}_{k} \rightarrow \hat{v}^{*} \quad \text { in } C_{l o c}^{\alpha}\left(\mathbb{R}^{n} \backslash\{0\}\right), \forall 0<\alpha<1 .
$$

By Theorem 1.2, $\hat{v}^{*}$ is radially symmetric. On the other hand, we deduce from (86) and (87) after passing to limit that

$$
\min _{|z|=1} \hat{v}^{*}(z) \leq 1+\epsilon, \quad \text { and } \max _{|z|=1} \hat{v}^{*}(z) \geq 1+2 \epsilon .
$$

The above violates the radial symmetry of $\hat{v}^{*}$. Proposition 4.1 is established.

\subsection{Detailed blow-up landscape}

The proof of Theorem 1.3 uses the following consequence of the Harnack-type inequality for conformally invariant equations, see $[4,7,16]$.

Lemma 4.6 Let $(f, \Gamma)$ satisfy (13)-(15) and (5). There exists a constant $C_{6}$, depending only on $(f, \Gamma)$, such that if $u \in C^{2}\left(B_{3}(0)\right)$ is a positive solution of

$$
f\left(\lambda\left(A^{u}\right)\right)=1 \text { in } B_{3}(0)
$$

then

$$
\int_{B_{1}(0)}|u|^{\frac{2 n}{n-2}} d x \leq C_{6} .
$$

Proof We give the proof here for completeness. By (5),

$$
-\Delta u \geq \frac{n-2}{2} \delta u^{\frac{n+2}{n-2}}>0 \text { in } B_{2}(0) .
$$

Thus, by Corollary A.2 in "Appendix A" as well as the maximum principle,

$$
\inf _{B_{2}(0)} u=\inf _{B_{2}(0) \backslash B_{3 / 2}(0)} u \geq \frac{1}{C} \int_{B_{1}(0)} u^{\frac{n+2}{n-2}} d x .
$$

It follows that

$$
\int_{B_{1}(0)} u^{\frac{2 n}{n-2}} d x \leq C \sup _{B_{1}(0)} u \inf _{B_{2}(0)} u .
$$

The conclusion follows from the above estimate and the Harnack-type inequality [8, Theorem 1.2]. (Note that (5) is used again here.)

Proof of Theorem 1.3 In view of Proposition 4.2 and (vi), it suffices to establish the theorem for $\epsilon=\epsilon_{0}:=1 / 2$.

By Lemma 4.6,

$$
\int_{B_{2}(0)} u^{\frac{2 n}{n-2}} d x \leq C_{6} .
$$

The constant $\bar{m}$ in the result can be selected to be the least integer satisfying

$$
\bar{m} \geq 2 C_{6}\left(\int_{B_{1}} U^{\frac{2 n}{n-2}} d x\right)^{-1} .
$$

(Clearly, this is an obvious upper bound for $m$ if the $x^{i}$,s satisfies (iii).) 
Let $\delta_{3}$ and $C_{3}$ be the constants in Lemma 4.4. Fix some $N_{0}>\frac{C_{1}}{\delta_{3}}$. Then there is some $r_{0} \in(3 / 2,2)$ such that

$$
\int_{r_{0}<|x|<r_{0}+\frac{1}{2 N_{0}}} u^{\frac{2 n}{n-2}} d x \leq \delta_{3} .
$$

By Lemma 4.4, this implies that

$$
u(x) \leq C_{3}\left(8 N_{0}\right)^{\frac{n-2}{2}}=: C_{7} \text { for all } r_{0}+\frac{1}{8 N_{0}}<|x|<r_{0}+\frac{3}{8 N_{0}} .
$$

Let $C_{0}$ and $\delta_{0}$ be as in Proposition 4.2 (corresponding to $\epsilon=\epsilon_{0}$ ). We can assume without loss of generality that

$$
C_{0}>2 \text { and } \delta_{0}<1 \text {. }
$$

We now declare

$$
C_{*}=\max \left(2 C_{7}, C_{0}\left(2 \delta_{0}^{-1}\right)^{\frac{\bar{m}(n-2)}{2}}\left(4 N_{0}\right)^{-\frac{n-2}{2}}\right) .
$$

This choice of $C_{*}$ will become clear momentarily.

Let $U_{1}=B_{r_{0}+\frac{3}{8 N_{0}}}(0)$ and $V_{1}=B_{r_{0}+\frac{1}{8 N_{0}}}(0) \subset U_{0}$. By (93), $C_{*} \geq 2 C_{7}$, and so, by (91), there is some $x^{1} \in V_{1} \subset B_{2}(0)$ such that

$$
u\left(x^{1}\right)=\sup _{U_{1}} u \geq C_{*} .
$$

Let $R_{1}=\frac{1}{4 N_{0}}$, then $(93)$ gives

$$
C_{*} \geq C_{0} R_{1}^{-\frac{n-2}{2}} .
$$

Hence, an application of Proposition 4.2 to $u$ on the ball $B_{R_{1}}\left(x^{1}\right)$ leads to

$$
\left|u(x)-U^{x^{1}, u\left(x^{1}\right)}(x)\right| \leq \epsilon_{0} U^{x^{1}, u\left(x^{1}\right)}(x), \quad \forall x \in B_{\delta_{0} R_{1}}\left(x^{1}\right) .
$$

In particular, for $\frac{\delta_{0} R_{1}}{2} \leq\left|x-x^{1}\right| \leq \delta_{0} R_{1}$,

$$
u(x) \leq 2 U^{x^{1}, u\left(x^{1}\right)}(x) \leq \frac{2}{u\left(x^{1}\right)\left|x-x^{1}\right|^{n-2}} \leq \frac{2^{n-1}}{C_{*}\left(\delta_{0} R_{1}\right)^{n-2}} \leq \frac{C_{*}}{2},
$$

where we have used (93) in the last estimate.

Let $U_{2}=U_{1} \backslash B_{\delta_{0}} R_{1} / 2\left(x^{1}\right)$ and $V_{2}=V_{1} \backslash B_{\delta_{0} R_{1}}\left(x^{1}\right) \subset U_{1}$. If

$$
\sup _{U_{2}} u \leq C_{*},
$$

we stop. Otherwise, in view of (94), there is some $x^{2} \in V_{2}$ such that

$$
u\left(x^{2}\right)=\sup _{U_{2}} u \geq C_{*} .
$$

We then let $R_{2}=\frac{\delta_{0} R_{1}}{2}$ so that (93) implies

$$
C_{*} \geq C_{0} R_{2}^{-\frac{n-2}{2}} .
$$

Hence, by Proposition 4.2,

$$
\left|u(x)-U^{x^{2}, u\left(x^{2}\right)}(x)\right| \leq \epsilon_{0} U^{x^{2}, u\left(x^{2}\right)}(x), \quad \forall x \in B_{\delta_{0} R_{2}}\left(x^{2}\right) .
$$


We then repeat the above process to define $U_{3}, V_{3}$, and to decide if a local max $x^{3}$ can be selected in $V_{3}$, etc. As explain above, the number $m$ of times this process can be repeated cannot exceed $\bar{m}$.

We have obtained the set of local maximum points $\left\{x^{1}, \ldots, x^{m}\right\}$ of $u$ and have verified (i) and (iv) for

$$
\delta_{*}=\left(\frac{\delta_{0}}{2}\right)^{\bar{m}} \frac{1}{2 N_{0}} \leq \delta_{0} R_{m}
$$

(vi) is readily seen as

$$
\operatorname{dist}\left(x^{i}, \partial U_{i}\right) \geq R_{i} \geq \delta_{*} .
$$

(ii) is also clear for

$$
K \geq\left(\frac{2}{\delta_{0}}\right)^{\bar{m}} 4 N_{0} \geq \frac{2}{\delta_{0} R_{m-1}} .
$$

From construction, we have

$$
\sup _{U_{m+1}} u \leq C_{*} .
$$

By Theorem 1.6, this implies that

$$
|\nabla \ln u(x)| \leq C_{8} \text { for all } x \in V_{m}=V_{0} \backslash \cup_{i=1}^{m} B_{\delta_{0} R_{i}}\left(x^{i}\right) .
$$

Also, note that, for $\delta_{*}<\left|x-x^{i}\right|<\delta_{0} R_{i}$, we have

$$
\frac{1}{u\left(x^{i}\right)}\left(\frac{1}{\left(C_{*}\right)^{-\frac{4}{n-2}}+\left(\delta_{0} R_{i}\right)^{2} .}\right)^{\frac{n-2}{2}} \leq U^{x^{i}, u\left(x^{i}\right)}(x) \leq \frac{1}{u\left(x^{i}\right)}\left(\delta_{*}\right)^{-(n-2)},
$$

and so

$$
\frac{1}{C_{9} u\left(x^{i}\right)} \leq u(x) \leq \frac{C_{9}}{u\left(x^{i}\right)}\left(C_{*}\right)^{-2}\left(\delta_{*}\right)^{-(n-2)}
$$

It is now clear that (iii) and (v) hold for $K$ sufficiently large. The proof is complete.

\section{A quantitative Liouville theorem}

Proof of Theorem 1.5 Assume by contradiction that, for some $\epsilon \in(0,1 / 2]$, there exist $v_{k} \epsilon$ $C^{2}\left(B_{3 R_{k}}(0), R_{k} \rightarrow \infty\right.$, such that $f\left(\lambda\left(A^{v_{k}}\right)\right)=1$ in $B_{3 R_{k}}(0)$ and $v_{k} \geq \gamma$ in $B_{r_{1}}(0)$ but, for each $k$,

$$
\text { (24) and (25) cannot simultaneously hold for any } \bar{x} \text {. }
$$

Define

$$
u_{k}(y)=R_{k}^{\frac{n-2}{2}} v_{k}\left(R_{k} y\right) \text { for }|y| \leq 3 .
$$

Then $f\left(\lambda\left(A^{u_{k}}\right)\right)=1$ in $B_{3}(0)$ and

$$
u_{k} \geq R_{k}^{\frac{n-2}{2}} \gamma \text { in } B_{r_{1} / R_{k}}(0) .
$$


Thus, by applying Theorem 1.4 and after passing to a subsequence, we can select sets of local maximum points $\left\{x_{k}^{1}, \ldots, x_{k}^{m}\right\}$ of $u_{k}$ such that assertions (i)-(vi) in Theorem 1.4 hold. We can also assume that $x_{k}^{i} \rightarrow x_{*}^{i}, 1 \leq i \leq m$.

By assertions (i), (iv) and (v) of Theorem 1.4, $u_{k}$ converges locally uniformly to zero in $B_{1} \backslash\left\{x_{*}^{1}, \ldots, x_{*}^{m}\right\}$. Thus, in view of (98), we must have $x_{*}^{i_{0}}=0$ for some (unique) $1 \leq i_{0} \leq m$. Clearly, $B_{r_{1} / R_{k}}(0) \subset B_{\delta_{*}}\left(x_{k}^{i_{0}}\right)$ for large $k$.

Recalling assertions (iv), (vi) and returning to the original sequence $v_{k}$ we get, for $\bar{x}_{k}=$ $R_{k} x_{k}^{i_{0}}$ and $\bar{\mu}_{k}=v_{k}\left(\bar{x}_{k}\right)=\sup _{B_{\delta_{*} R_{k}}\left(\bar{x}_{k}\right)} v_{k}$, that $B_{r_{1}}(0) \subset B_{\delta_{*} R_{k}}\left(\bar{x}_{k}\right), \bar{\mu}_{k} \geq \gamma$ and

$$
(1-\epsilon) U^{\bar{x}_{k}, \bar{\mu}_{k}} \leq v_{k} \leq(1+\epsilon) U^{\bar{x}_{k}, \bar{\mu}_{k}} \text { in } B_{\delta_{*} R_{k}}\left(\bar{x}_{k}\right) .
$$

We then have

$$
\gamma \leq v_{k}(0)=2\left(\frac{\bar{\mu}_{k}^{\frac{2}{n-2}}}{1+\bar{\mu}_{k}^{\frac{4}{n-2}}\left|\bar{x}_{k}\right|^{2}}\right)^{\frac{n-2}{2}} \leq \frac{2}{\bar{\mu}_{k}\left|\bar{x}_{k}\right|^{n-2}} \leq \frac{2}{\gamma\left|\bar{x}_{k}\right|^{n-2}}
$$

This implies

$$
\left|\bar{x}_{k}\right| \leq 2^{\frac{1}{n-2}} \gamma^{-\frac{2}{n-2}} .
$$

On the other hand, since $B_{r_{1}}(0) \backslash B_{r_{1} / 2}\left(\bar{x}_{k}\right) \neq \emptyset$, we can select some $y_{k} \in B_{r_{1}}(0)$ such that

$$
\left|\bar{x}_{k}-y_{k}\right| \geq \frac{r_{1}}{2} \text {. }
$$

This implies that

$$
\gamma \leq v\left(y_{k}\right) \leq 2 U^{\bar{x}_{k}, \bar{\mu}_{k}}\left(y_{k}\right) \leq \frac{2}{\bar{\mu}_{k}\left|y_{k}-\bar{x}_{k}\right|^{n-2}} \leq \frac{2^{n-1}}{\bar{\mu}_{k} r_{1}^{n-2}},
$$

and so

$$
\bar{\mu}_{k} \leq \frac{2^{n-1}}{\gamma r_{1}^{n-2}} .
$$

We have thus shown that $\bar{x}=\bar{x}_{k}$ satisfies both (24) and (25), which contradicts (97).

Open Access This article is distributed under the terms of the Creative Commons Attribution 4.0 International License (http://creativecommons.org/licenses/by/4.0/), which permits unrestricted use, distribution, and reproduction in any medium, provided you give appropriate credit to the original author(s) and the source, provide a link to the Creative Commons license, and indicate if changes were made.

\section{Appendix A: A remark on positive superharmonic functions}

Let $G(x, y)$ be the Green's function of $-\Delta$ in $B_{1} \backslash B_{\rho} \subset \mathbb{R}^{n}, 0<\rho<1 / 2$ :

$$
G(x, y):=\frac{1}{n(n-2) \alpha(n)}\left(\frac{1}{|x-y|^{n-2}}-h(x, y)\right), \quad x, y \in B_{1} \backslash B_{\rho},,
$$

where $\alpha(n)$ denotes the volume of the unit ball in $\mathbb{R}^{n}$, and $h(x, y)$ satisfies, for $x \in B_{1} \backslash B_{\rho}$,

$$
\left\{\begin{array}{l}
-\Delta_{y} h(x, y)=0, \quad y \in B_{1} \backslash B_{\rho}, \\
h(x, y)=\frac{1}{|x-y|^{n-2}}, y \in \partial\left(B_{1} \backslash B_{\rho}\right) .
\end{array}\right.
$$


Lemma A.1 For any $0<2 \rho<\rho_{0}<\rho_{1}<\rho_{2}<1$, there exists some constants $C, C^{\prime}>1$, depending only on $n, \rho_{0}, \rho_{1}, \rho_{2}$, such that the Green's function $G$ for $B_{1} \backslash B_{\rho}$ satisfies

$$
G(x, y) \geq \frac{1}{C}\left(1-\frac{\rho^{n-2}}{|y|^{n-2}}\right), \quad \forall \rho \leq|y| \leq \rho_{0}, \quad \rho_{1} \leq|x| \leq \rho_{2} .
$$

Consequently,

$$
G(x, y) \geq 1 / C^{\prime}, \quad \forall 2 \rho \leq|y| \leq \rho_{0}, \rho_{1} \leq|x| \leq \rho_{2} .
$$

Proof In the following we use $C_{1}, C_{2}, \ldots$ to denote positive constants depending only on $\rho_{0}, \rho_{1}, \rho_{2}$ and $n$. For a fixed $x$ satisfying $\rho_{1} \leq|x| \leq \rho_{2}$, it follows from the maximum principle that for some positive constant $C_{1}$,

$$
0<h(x, y) \leq C_{1}, \quad \forall y \in B_{1} \backslash B_{\rho} .
$$

It follows that for some positive constants $C_{2}$ and $C_{3}$,

$$
G(x, y) \geq|x-y|^{2-n}-C_{1} \geq 1 / C_{2}, \quad \forall y \in B_{2 / C_{3}}(x) \backslash\{x\} \subset B_{1} \backslash B_{\rho_{0}} .
$$

Since $G(x, y)$ is a positive harmonic function of $y$ in $\left(B_{1} \backslash B_{\rho}\right) \backslash\{x\}$. We can apply the Harnack inequality to obtain, for some $C_{4}$,

$$
G(x, y) \geq 1 / C_{4}, \quad \forall|y|=\rho_{0} .
$$

By the maximum principle,

$$
G(x, y) \geq \frac{1}{C_{4}}\left(1-\frac{\rho^{n-2}}{|y|^{n-2}}\right), \quad \rho<|y| \leq \rho_{0} .
$$

It follows that for some $K$,

$$
G(x, y) \geq 1 / K, \quad 2 \rho \leq|y| \leq \rho_{0} .
$$

Lemma A.1 is established.

Corollary A.1 For any $0<2 \rho<\rho_{0}<\rho_{1}<\rho_{2}<1$, let

$$
\left\{\begin{array}{rlrl}
-\Delta \tilde{v}=\tilde{f} \geq 0, & & B_{1} \backslash B_{\rho}, \\
\tilde{v} & \geq 0, & & \partial\left(B_{1} \backslash B_{\rho}\right) .
\end{array}\right.
$$

Then, for some constants $C, C^{\prime}>1$ depending only on $n, \rho_{0}, \rho_{1}, \rho_{2}$,

$$
\inf _{\rho_{1} \leq|x| \leq \rho_{2}} \tilde{v}(x) \geq \frac{1}{C} \int_{\rho \leq|y| \leq \rho_{0}}\left(1-\frac{\rho^{n-2}}{|y|^{n-2}}\right) \tilde{f}(y) d y \geq \frac{1}{C^{\prime}} \int_{2 \rho \leq|y| \leq \rho_{0}} \tilde{f}(y) d y .
$$

Proof For $\rho_{1} \leq|x| \leq \rho_{2}$, we use the Green's formula to obtain

$$
\tilde{v}(x)=\int_{B_{1} \backslash B_{\rho}} G(x, y) \tilde{f}(y) .
$$

Corrollary A.1 follows from Lemma A.1. 
Corollary A.2 For any $0<\rho_{0}<\rho_{1}<\rho_{2}<1$, let

$$
\left\{\begin{aligned}
-\Delta \tilde{v}=\tilde{f} \geq 0, & B_{1}, \\
\tilde{v} \geq 0, & \partial B_{1} .
\end{aligned}\right.
$$

Then, for some constants $C$ depending only on $n, \rho_{0}, \rho_{1}, \rho_{2}$,

$$
\inf _{\rho_{1} \leq|x| \leq \rho_{2}} \tilde{v}(x) \geq \frac{1}{C} \int_{|y| \leq \rho_{0}} \tilde{f}(y) d y .
$$

Proof This follows from Corollary A.1 by sending $\rho \rightarrow 0$.

Corollary A.3 For $0<r<R / 2$, let

$$
\left\{\begin{aligned}
-\Delta v=f \geq 0, & & B_{R} \backslash B_{r} \\
v \geq 0, & & \partial\left(B_{R} \backslash B_{r}\right) .
\end{aligned}\right.
$$

Then, for any $\frac{2 r}{R}<\rho_{0}<\rho_{2}<\rho_{2}<1$, there exist some constants $C, C^{\prime}>1$ depending only on $n, \rho_{0}, \rho_{1}, \rho_{2}$,

$$
\begin{aligned}
\inf _{\rho_{1} R \leq|x| \leq \rho_{2} R} v(x) & \geq \frac{1}{C R^{n-2}} \int_{r \leq|y| \leq \rho_{0} R}\left(1-\frac{r^{n-2}}{|y|^{n-2}}\right) f(y) d y \\
& \geq \frac{1}{C^{\prime} R^{n-2}} \int_{2 r \leq|y| \leq \rho_{0} R} f(y) d y .
\end{aligned}
$$

Proof Performing a change of variables

$$
\tilde{v}(x):=R^{n-2} v(R x), \quad \tilde{f}(x):=R^{n} f(R x), \rho=r / R,
$$

we obtain Corollary A.3 from Corollary A.1

\section{Appendix B: A remark on viscosity solutions}

In this section we consider the convergence of viscosity solutions in a slightly more general context. Let $\mathbb{R}^{n \times n}, S y m^{n \times n}, S y m_{+}^{n \times n}$ denote the set of $n \times n$ matrices, symmetric matrices, and positive definite symmetric matrices, respectively. Let $\mathcal{M}=\mathbb{R}^{n \times n}$ or $\mathcal{M}=S y m^{n \times n}$. Let $\Omega \subset \mathbb{R}^{n}, U \subset \mathcal{M}$ be open, $F \in C(U), A \in C\left(\Omega \times \mathbb{R} \times \mathbb{R}^{n} \times S y m^{n \times n} ; \mathcal{M}\right)$ and consider partial differential equations for the form

$$
F\left(A\left(x, u, \nabla u, \nabla^{2} u\right)\right)=0 .
$$

To keep the notation simple, we will abbreviate $A[u]=A\left(\cdot, u, \nabla u, \nabla^{2} u\right)$, and whenever we write $F(M)$, we implicitly assume that $M \in U$.

In applications, it is frequently assumed that

$$
\begin{aligned}
& M+N \in U \text { for } M \in U \text { and } N \in S y m_{+}^{n \times n}, \\
& F(M+N) \geq F(M) \text { for } M \in U \text { and } N \in S y m_{+}^{n \times n}, \\
& A(x, z, p, M)-A(x, z, p, M+N) \text { is non-negative definite for } N \in S y m_{+}^{n \times n} .
\end{aligned}
$$

In the main body of the paper,

$$
\begin{aligned}
\mathcal{M} & =S y m^{n \times n}, U \text { is the set of symmetric matrices such that } \lambda(U) \in \Gamma, \\
F(M) & =f(\lambda(M))-1, \text { and } A[v]=A^{v} .
\end{aligned}
$$


The following definition is "consistent" with the assumptions (99), (100) and (101) and with Definition 1.1.

Definition B.1 A continuous function $v$ is an open set $\Omega \subset \mathbb{R}^{n}$ is a viscosity supersolution (respectively, subsolution) of

$$
F(A[v])=0, \text { in } \Omega,
$$

when the folowing holds: if $x_{0} \in \Omega, \varphi \in C^{2}(\Omega),(v-\varphi)\left(x_{0}\right)=0$, and $v-\varphi \geq 0$ near $x_{0}$, then

$$
F\left(A[\varphi]\left(x_{0}\right)\right) \geq 0 .
$$

(respectively, if $(v-\varphi)\left(x_{0}\right)=0$, and $v-\varphi \leq 0$ near $x_{0}$, then either $A[\varphi]\left(x_{0}\right) \notin U$ or $\left.F\left(A[\varphi]\left(x_{0}\right)\right) \leq 0\right)$. Equivalently, we write $F(A[v]) \geq 0$ (respectively, $F(A[v]) \leq 0$ ) in $\Omega$ in the viscosity sense.

We say that $v$ is a viscosity solution if it is both a viscosity supersolution and a viscosity subsolution.

Proposition B.1 Let $\Omega \subset \mathbb{R}^{n}, U \subset \mathcal{M}$ be open, $F \in C(U), A \in C\left(\Omega \times \mathbb{R} \times \mathbb{R}^{n} \times\right.$ $\left.S_{y m}{ }^{n \times n} ; \mathcal{M}\right)$ and assume that the structural conditions (99), (100) and (101) are in effect.

(a) If $v_{k}$ satisfies $F\left(A\left[v_{k}\right]\right) \leq 0$ in $\Omega$ in the viscosity sense and if $v_{k}$ converges in $C_{l o c}^{0}(\Omega)$ to $v$, then $v$ also satisfies $F(A[v]) \leq 0$ in $\Omega$ in the viscosity sense.

(b) Assume further that the closure of $\bar{F}^{-1}([0, \infty))$ in $\mathcal{M}$ is a subset of $U$, i.e.

$$
\text { if } A_{k} \in U, F\left(A_{k}\right) \geq 0 \text { and } A_{k} \rightarrow A \text { as } k \rightarrow \infty \text {, then } A \in U \text {. }
$$

If $v_{k}$ satisfies $F\left(A\left[v_{k}\right]\right) \geq 0$ in $\Omega$ in the viscosity sense and if $v_{k}$ converges in $C_{\text {loc }}^{0}(\Omega)$ to $v$, then $v$ also satisfies $F(A[v]) \geq 0$ in $\Omega$ in the viscosity sense.

Remark B.1 In general, condition (103) cannot be dropped in (b).

As a first example, consider

$$
\begin{aligned}
F(M) & =\operatorname{tr}(M), \quad U=\left\{M \in \operatorname{Sym}^{n \times n}: \operatorname{tr}(M)>0\right\}, \\
A[v] & =-\nabla^{2} v .
\end{aligned}
$$

Clearly, (99), (100) and (101) are satisfied. Now, if $v_{k}=-\frac{1}{k}|x|^{2}$, then $A\left[v_{k}\right] \in U$ but $v=\lim _{k \rightarrow \infty} v_{k}=0$ does not satisfy $A[v] \in U$.

The situation does not improve even if one imposes that $v_{k}$ is a solution and that $U$ is a maximal set where the ellipticity condition (100) holds. See Remark 2.1.

Remark B.2 If $(f, \Gamma)$ satisfies (1)-(4) and if $(F, U)$ are given by (102), then all hypotheses of Proposition B.1 are met. In particular if $f\left(\lambda\left(A^{v_{k}}\right)\right)=1$ in some open set $\Omega \subset \mathbb{R}^{n}$ in the viscosity sense and $v_{k}$ converges in $C_{l o c}^{0}(\Omega)$ to $v$, then $f\left(\lambda\left(A^{v}\right)\right)=1$ in $\Omega$ in the viscosity sense.

Proof The proof is standard and we include it here for readers' convenience. We will only show (b). The proof of (a) is similar. Fix $x_{0} \in \Omega$ and assume that $\varphi \in C^{2}(\Omega)$ such that $(v-$ $\varphi)\left(x_{0}\right)=0$ and $v-\varphi \geq 0$ in some small ball $B_{\rho}\left(x_{0}\right)$. We need to show that $F\left(A[\varphi]\left(x_{0}\right)\right) \geq 0$.

For $\delta \in(0, \rho)$, let $\varphi_{\delta}(x)=\varphi(x)-\delta\left|x-x_{0}\right|^{2}$. Since $\varphi_{\delta}(x)=\varphi(x)-\delta^{3} \leq v(x)-\delta^{3}$ for $\left|x-x_{0}\right|=\delta$, the convergence of $v_{k}$ to $v$ implies that

$$
v_{k}(x) \geq \varphi_{\delta}(x)+\frac{1}{2} \delta^{3} \text { for all }\left|x-x_{0}\right|=\delta \text { and all sufficiently large } k .
$$


Thus, as $\min _{B_{\delta}\left(x_{0}\right)}\left(v-\varphi_{\delta}\right)=0$, there is some $x_{k} \in B_{\delta}\left(x_{0}\right)$ such that

$$
\beta_{k}:=\left(v_{k}-\varphi_{\delta}\right)\left(x_{k}\right)=\min _{\bar{B}_{\delta}\left(x_{0}\right)}\left(v_{k}-\varphi_{\delta}\right) \rightarrow 0 \text { as } k \rightarrow \infty .
$$

Also, since

$$
\beta_{k}=\left(v_{k}-\varphi\right)\left(x_{k}\right)+\delta\left|x_{k}-x_{0}\right|^{2} \geq\left(v_{k}-v\right)\left(x_{k}\right)+\delta\left|x_{k}-x_{0}\right|^{2},
$$

we have that $x_{k} \rightarrow x_{0}$ as $k \rightarrow \infty$.

Now since $\hat{\varphi}_{k}:=\varphi_{\delta}+\beta_{k}$ satisfies $\left(v_{k}-\hat{\varphi}_{k}\right)\left(x_{k}\right)=0, v_{k}-\hat{\varphi}_{k} \geq 0$ in $B_{\delta}\left(x_{0}\right)$, and since $v_{k}$ is a solution of $F\left(A\left[v_{k}\right]\right)=0$, we infer that

$$
F\left(A\left[\hat{\varphi}_{k}\right]\left(x_{k}\right)\right) \geq 0 .
$$

Sending $k \rightarrow \infty$ and then $\delta \rightarrow 0$ and using (103), we deduce that $A[\varphi]\left(x_{0}\right) \in U$ and

$$
F\left(A[\varphi]\left(x_{0}\right)\right) \geq 0,
$$

as desired. (Note that (103) is not required to show that (a).)

\section{Appendix C: A calculus lemma}

We collect here a couple calculus statements which was used when we applied the method of moving spheres in the body of the paper.

Lemma C.1 Let $f \in C^{0}\left(\mathbb{R}^{n} \backslash\{0\}\right), n \geq 1, v>0$. Assume that, for all $x \in \mathbb{R}^{n}$ and $0<\lambda \leq$ $|x|$, there holds

$$
\left(\frac{\lambda}{|y-x|}\right)^{v} f\left(x+\frac{\lambda^{2}(y-x)}{|y-x|^{2}}\right) \leq f(y) \text { for } y \in \mathbb{R}^{n} \backslash\left(B_{\lambda}(x) \cup\{0\}\right) .
$$

Then $f$ is radially symmetric about the origin and $f:(0, \infty) \rightarrow(0, \infty)$ is non-increasing.

Proof This was established in [10]. See the argument following equation (110) therein.

Corollary C.1 Let $f \in C^{0}\left(\mathbb{R}^{n}\right), n \geq 1, v>0$. Assume that

$$
\left(\frac{\lambda}{|y-x|}\right)^{\nu} f\left(x+\frac{\lambda^{2}(y-x)}{|y-x|^{2}}\right) \leq f(y), \quad \forall \lambda>0, x \in \mathbb{R}^{n},|y-x| \geq \lambda .
$$

Then $f \equiv$ constant.

\section{References}

1. Caffarelli, L., Gidas, B., Spruck, J.: Asymptotic symmetry and local behavior of semilinear elliptic equations with critical Sobolev growth. Commun. Pure Appl. Math. 42, 271-297 (1989)

2. Caffarelli, L., Nirenberg, L., Spruck, J.: The Dirichlet problem for nonlinear second-order elliptic equations. III. Functions of the eigenvalues of the Hessian. Acta Math. 155, 261-301 (1985)

3. Chang, S.-Y.A., Gursky, M.J., Yang, P.: Entire solutions of a fully nonlinear equation. In: Lectures on Partial Differential Equations, Vol. 2 of New Studies in Advanced Mathematics, pp. 43-60. International Press, Somerville (2003)

4. Chen, C.-C., Lin, C.-S.: Estimates of the conformal scalar curvature equation via the method of moving planes. Commun. Pure Appl. Math. 50, 971-1017 (1997) 
5. Gidas, B., Ni, W.M., Nirenberg, L.: Symmetry of positive solutions of nonlinear elliptic equations in $\mathbf{R}^{n}$. In: Mathematical Analysis and Applications, Part A, Vol. 7 of Advanced in Mathematical Supply Studies, pp. 369-402. Academic Press, New York (1981)

6. Guan, P., Wang, G.: Local estimates for a class of fully nonlinear equations arising from conformal geometry. Int. Math. Res. Not. 26, 1413-1432 (2003)

7. Li, A., Li, Y.Y.: On some conformally invariant fully nonlinear equations. Commun. Pure Appl. Math. 56, 1416-1464 (2003)

8. Li, A., Li, Y.Y.: On some conformally invariant fully nonlinear equations. II. Liouville, Harnack and Yamabe. Acta Math. 195, 117-154 (2005)

9. Li, Y.Y.: Prescribing scalar curvature on $S^{n}$ and related problems. I. J. Differ. Equ. 120, 319-410 (1995)

10. Li, Y.Y.: Conformally invariant fully nonlinear elliptic equations and isolated singularities. J. Funct. Anal. 233, 380-425 (2006)

11. Li, Y.Y.: Degenerate conformally invariant fully nonlinear elliptic equations. Arch. Ration. Mech. Anal. 186, 25-51 (2007)

12. Li, Y.Y.: Local gradient estimates of solutions to some conformally invariant fully nonlinear equations. Commun. Pure Appl. Math. 62, 1293-1326 (2009). (C. R. Math. Acad. Sci. Paris 343 (2006), no. 4, 249-252)

13. Li, Y.Y., Nguyen, L.: Harnack inequalities and Bôcher-type theorems for conformally invariant, fully nonlinear degenerate elliptic equations. Commun. Pure Appl. Math. 67, 1843-1876 (2014)

14. Li, Y.Y., Zhang, L.: Liouville-type theorems and Harnack-type inequalities for semilinear elliptic equations. J. Anal. Math. 90, 27-87 (2003)

15. Li, Y.Y., Zhu, M.: Uniqueness theorems through the method of moving spheres. Duke Math. J. 80, 383-417 (1995)

16. Schoen, R.: Courses at Stanford University, 1988, and New York University (1989) 